\title{
Long-period comets with non-gravitational effects ${ }^{\star}$
}

\author{
M. Królikowska
}

\begin{abstract}
Space Research Centre of the Polish Academy of Sciences, Bartycka 18A, 00-716, Warsaw, Poland e-mail: mkr@cbk.waw.pl
\end{abstract}

Received 24 May 2004 / Accepted 24 June 2004

\begin{abstract}
The influence of non-gravitational effects on the motion of long-period comets is analyzed. Investigations were performed for about 60 nearly-parabolic comets discovered during the last thirty years. All three non-gravitational parameters $A_{1}, A_{2}, A_{3}$ were detected in the motion of 19 comets on the basis of positional observations only. For these comets the standard $g(r)$ function as well as some of its modifications were used to model the ice sublimation from the cometary nucleus. For 14 of 19 comets the orientation of spin axes of the nucleus were also determined. Finally, the magnitude of the nongravitational effects derived for the long-period comets have been compared with observed the non-gravitational perturbations on periodic comets.
\end{abstract}

Key words. solar system: general - comets: general

\section{Introduction}

It is well known that non-gravitational (NG) effects play an essential role in the motion of almost all the short-period comets. The NG solutions have been derived by studying several successive apparitions. Contrary to the short-period comets, the NG orbits have been determined for a very small fraction of the known long-period comets. The first detections of the NG forces in the motion of these comets were reported by Marsden and collaborators in the late 1960s and early 1970s (Marsden 1969; 1970; Marsden et al. 1973). In the Catalogue of Cometary Orbits (Marsden \& Williams 2003, hereafter MW Catalogue or MWC) only 23 of 400 nearly parabolic comets have detectable radial and transverse components of the NG force. The problem is obviously observational: the long-period comets are observed on one passage through the inner Solar System. In practice, it turns out that the potential candidates for the detectable NG effects should be observed several months before and after their perihelion passage.

The NG effects are also important for an identification of hyperbolic comets. The problem of a negative tail in the distribution of the reciprocals of the original semimajor axis $\left(1 / a_{\text {ori }}\right)$ has been widely discussed in the literature. Marsden et al. (1973) speculated that the number of hyperbolic original orbits could be overestimated due to omission of the NG effects. In the 1990s, Yabushita (1991) and Bolatto et al. (1995) considered the NG perturbation in a comet's energy per orbital revolution. They concluded that these perturbations are too small to explain the negative excess of the original binding energy of "hyperbolic" comets. I have investigated this problem in a

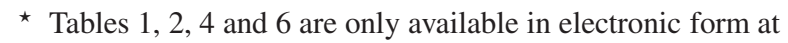
http://www. edpsciences.org separate paper (Królikowska 2001, hereafter Paper I) where the sample of 33 comets considered as "hyperbolic" in the sense of a pure gravitational orbit were analyzed; the radial and transverse components of the NG accelerations were derived for 16 of them. It was shown that misleading results are obtained when the same osculating orbit is used as the initial orbit for backwards integrations using the NG effects and without these effects. The differences in the resulting original reciprocals are in this case significantly smaller than $10^{-4} \mathrm{AU}^{-1}$. The respective differences between two original reciprocal semimajor axes increase even to about $3 \times 10^{-3} \mathrm{AU}^{-1}$ when the NG osculating orbit and pure gravitational osculating orbit are separately used as the initial orbit for the backwards integrations. Therefore, for 14 of 16 comets with the detectable NG effects, the original orbits have been changed from hyperbolic to elliptic, and for the remaining two comets the original orbits became less hyperbolic as a results of the NG orbit solution. This tendency to make $1 / a_{\text {ori }}$ positive is generated by the small changes in the orbital osculating eccentricity derived from the positional observations when the NG effects are taken into consideration.

In the present paper the NG motions of individual nearly parabolic comets from the MW Catalogue are carefully examined. Two famous long-period comets, C/1995 O1 Hale-Bopp and C/1996 B2 Hyakutake are included in this investigation for comparison with the remaining comets. A more detailed analysis of the motion of these two spectacular objects is described by Szutowicz et al. (2002a,b).

There are important reasons to speculate that the standard $g(r)$-function could be non-representative for the modelling the NG accelerations detected in the motion of the long-period comets. First, the application of the standard $g(r)$ determined from the photometric studies of the short-period comets to the 
long-period ones is based on the assumption of the similarity between these two comet families. It is also implicitly assumed that the long-period comets constitute a uniform class of objects. In the third article of his classic series on the NG effects, Marsden (1970) derived the solutions for two long-period comets C/1959 Y1 Burnham and C/1956 R1 Arend-Roland according to his style I NG model. He concluded that the NG forces acting on these two comets "seem to obey something like an inverse-square law, whereas the forces on short-period comets definitely do not". Recently, the detailed studies of C/1995 O1 Hale-Bopp and C/1996 B2 Hyakutake (Szutowicz et al. 2002a,b) show that the $g(r)$-like forms based on the photometric data are very different for these two objects, and significantly depart from the inverse-square law. The next problem is related to the unacceptably large values of the standard NG parameter $A_{1}$ determined from the observations. This problem also has been known for a long time (Whipple 1977; Yabushita 1991, 1996).

The standard function $g(r)$ is still used to determine the NG effects in the motion of the long-period comets because the relevant function has not yet been found. One should stress that there are strong arguments for the reality of calculated NG forces. First, the rms of the fitted NG orbits to the positional data are substantially reduced (Table 1). Second, the positive value of the radial parameter $A_{1}$ determined for all the comets from the sample (MW Catalogue and Table 2) indicate that the NG forces are directed radially away from the Sun. The "vent" model working also with the negative values of $A_{1}$, seems to be inapplicable to those long-period comets that have made only a few passages through the inner solar system in their dynamical history. Next, the inclusion of the NG effects in orbit determinations for dynamically new comets cancels the apparent hyperbolicity of their orbits, as has been already discussed (Marsden et al. 1973; Królikowska, Paper I).

The aim of this investigation was to analyze the NG effects based on different forms of NG acceleration as a function of the cometary heliocentric distance.

\section{Selection of the long-period comets}

The preliminary sample of nearly-parabolic comets selected from the MW Catalogue contains the following objects:

- all comets observed after the year 1970 (which guarantees a good quality of positional data) whose observational arc covered more than half a year time interval;

- selected comets discovered after 1955: only with determined NG parameters $A_{1}$ and $A_{2}$.

Marsden \& Williams (2001) listed 18 comets with known $A_{1}$ and $A_{2}$ in their catalogue. In the present investigation we have found that the normal component of the NG force, $A_{3}$, is determinable for 12 of them.

The original catalogue orbits of comets selected according to these criteria are hyperbolic (20 objects) or elliptical (41 objects). The problem of hyperbolic incoming orbits for single-apparition comets was discussed earlier (Paper I) where all the 33 comets suspected by Marsden and Williams of hyperbolicity were investigated. As was mentioned, 16 comets of those 33 objects have detectable NG effects. For only five of them (C/1959 Y1 Burnham, C/1986 P1A Wilson, C/1995 Y1 Hyakutake, C/1998 P1 Williams and C/1996 E1 NEAT) was the accuracy of all three NG parameters sufficient for the detailed analysis of their NG effects. Four of them are listed in the MW Catalogue as comets with known $A_{1}$ and $A_{2}$.

For the first time the NG effects were determined for six comets among 41 objects with incoming elliptical orbits: C/1975 T1, C/1987 W2, C/1991 B1, C/1991 T1, C/1999 H1, C/1999 J3.

Thus, the final sample consisting of 19 comets with NG effects was investigated in detail (Table 1). Two prominent members of this sample, Comet C/1995 O1 Hale-Bopp and Comet C/1996 B2 Hyakutake, have been treated elsewhere (Szutowicz et al. 2002a,b). Here, these two famous comets are discussed for comparison with the remaining long-period comets with orbits affected by NG forces.

\section{The orbit determinations}

The orbital elements of each comet were recomputed using the archive observations available at the Minor Planet Center (Cambridge, USA). The positional data of comet C/1959 Y1 Burnham were additionally supplemented by 51 observations found in the literature eliminating potential bias which sometimes affects data obtained by a single observer. The observations for individual comets were selected according to the objective criteria elaborated by Bielicki \& Sitarski (1991). Table 1 contains characteristics of the observational material for all the considered comets.

In almost all the cases the rms based on all the observations are significantly larger than the rms obtained on the basis of pre- or post-perihelion observations alone. In the numerical calculations it is the first sign that NG forces are detected in the comet's motion. When the NG force was included in the cometary equation of motion the rms dropped significantly, see Col. 7 of Table 1. The listed values of the rms represent the NG solutions based on the standard $g(r)$ function (see the next Section). The most spectacular reduction of the rms was obtained for comets C/1998 P1 Williams (from 4". 58 to 1'. 10) and C/1993 A1 Mueller (from 2".79 to 1". 14), the smallest drop - for Comet C/1991 T2 Shoemaker-Levy (by 0.'07). However, only three observations of $1991 \mathrm{~T} 2$ were made within a month after the perihelion passage, and this could explain the small change of the rms.

To calculate the original and future reciprocals of semimajor axes, each comet was followed from its position at a given epoch backwards (orbit before planetary perturbations) and forwards (outgoing orbit) until the comet reached a distance of $250 \mathrm{AU}$ from the Sun. The equations of motion have been integrated in barycentric coordinates using the recurrent power series method (Sitarski 1989, 2002) taking into account the perturbations by all nine planets. All numerical calculations presented here are based on the Warsaw ephemeris DE405/WAW, i.e. the numerical Solar System ephemeris consistent to high accuracy with the JPL ephemeris DE405 (Sitarski 2002).

The respective values of original and future reciprocals of semimajor axes are given in the last two columns of 
Table 1 . It is interesting to compare values of $1 / a_{\text {ori }}$ and $1 / a_{\text {fut }}$ for the five comets (1959 Y1 Burnham, 1998 P1 Williams, 1996 P1A Wilson, 1996 E1 NEAT and 1993 A1 Mueller) considered in the previous paper (Paper I). It was shown that the NG effects (described by two standard parameters $A_{1}$ and $A_{2}$ ) are responsible for small modifications of the pure gravitational osculating elements. These minute modifications are sufficient, however, to obtain the elliptic original orbits for comets recognized in the literature as hyperbolic. The present numerical calculations are based on different Solar System ephemerides and include also the normal component of the NG force, $A_{3}$. Thus, $1 / a_{\text {ori }}$ and $1 / a_{\text {fut }}$ derived here for the NG orbits differ slightly from that given in Table 1 of Paper I. Nevertheless, the same tendency is evident. For two comets listed in Table 1, C/1995 Y1 Hyakutake and C/1959 Y1 Burnham, the original NG orbits seem be more hyperbolic than the original gravitational orbits.

In the case of $\mathrm{C} / 1995 \mathrm{Y} 1$ the original $\mathrm{NG}$ orbit defined by $1 / a_{\text {ori }}=-67 \pm 60$ (in the units of $10^{-6} \mathrm{AU}^{-1}$ used in this paper) is slightly more hyperbolic than the pure gravitational orbit characterized by $1 / a_{\text {ori }}=-4 \pm 6$, where uncertainties of $1 / a_{\text {ori }}$ are based on the statistical approach to the orbit determinations (Paper I). In the previous investigations this object had a slightly elliptical original orbit $1 / a_{\text {ori }}=+4 \pm 6$ $\left(1 / a_{\text {ori }}=+11 \pm 10\right.$ for NG orbit $)$ and was not classified as a candidate hyperbolic comet. However, it will be shown that a different form of the NG function rather than the standard $g(r)$ function should be used to adequately describe the NG motion of this comet. The absolute value of the transverse component $A_{2}$ given in the MW Catalogue for C/1995 Y1 is greater than the radial component $A_{1}$; this also suggests another form of the NG function than the standard $g(r)$.

The elipticity of the original orbit of the second object, comet C/1959Y1 Burnham, is also uncertain. The NG motion of this comet has been carefully investigated by many researchers in the past (van Biesbroeck \& Marsden 1963; Sekanina 1968; Matese et al. 1992). It was known as one of only a few dynamically new comets that had original gravitational orbit nominally hyperbolic at a level of $5 \sigma$ of the formal measured error (Matese et al. 1993; Marsden et al. 1978). In Paper I only 37 measurements by Roemer et al. (1966) were taken into account. The $1 / a_{\text {ori }}$ for the NG orbit was then positive and equaled $+188 \pm 50$, whereas the original gravitational orbit was hyperbolic with $1 / a_{\text {ori }}=-135 \pm 26$. When 88 positional observations (covering the same six-month interval as in Roemer et al.) is used the $1 / a_{\text {ori }}$ are $-139 \pm 17$ and $-202 \pm 101$ for the gravitational and NG orbits, respectively. This example illustrates that actual uncertainties in some cases could exceed the formal errors of $1 / a_{\text {ori }}$. Therefore, it is more advisable to discuss tendencies of the NG orbit to be more eliptic or more hyperbolic in comparison to the gravitational one (see also Paper I). It will be shown, however, that assumption of asymmetric NG acceleration leads to the positive value of $1 / a_{\text {ori }}$ for this object (Sect. 5).

The values of $1 / a_{\text {ori }}$ (Table 1 ) provide the information of which comets are dynamically new. From the observed Oort peak of comets we obtain the canonical rough requirement
$1 / a_{\text {ori }}<10^{-4} \mathrm{AU}^{-1}$ for orbits of comets coming from the Oort cloud. Since most of the comets in the Oort spike are on their first passage through the inner part of the Solar System, such comets may contain plenty of volatile ices $\left(\mathrm{CO}, \mathrm{CO}_{2}\right.$, etc.), which should be taken into account in the modelling of the NG effects. From this point of view seven comets could be dynamically new: C/1959 Y1 Burnham, C/1990 K1 Levy, C/1995 Y1 Hyakutake, C/1998 P1 Williams, C/1996 P1A Wilson, C/1996 E1 NEAT, and C/1993 A1 Mueller. That three of these objects probably will escape from the Solar System.

\section{Non-gravitational models of cometary outgassing}

The term "non-gravitational effect" has been reserved for the outgassing acceleration of the comet nucleus caused by anisotropic sublimation of volatiles from the cometary nucleus. The classical method to determine the NG effects on the orbital motion of a comet was proposed by Marsden et al. (1973). Their commonly-used style II model assumes that accelerations are symmetric about perihelion, and can be expressed by the semiempirical function $g(r)$ :

$F_{i}=A_{i} \cdot g(r), \quad A_{\mathrm{i}}=$ const. for $i=1,2,3$,

where $F_{1}, F_{2}, F_{3}$ represent the radial, transverse and normal components of the NG acceleration, respectively.

The form of the dimensionless function $g(r)$ is related to the empirical water sublimation curve derived by Delsemme \& Miller (1971), and simulates the ice sublimation rate as a function of the heliocentric distance $r$ :

$\mathrm{g}(r)=\alpha\left(r / r_{0}\right)^{-m}\left[1+\left(r / r_{0}\right)^{n}\right]^{-k}, \quad$ where

$m=2.15, \quad n=5.093, \quad k=4.6142$

$\alpha=0.1113 \quad r_{0}=2.808 \mathrm{AU}$.

The normalization constant $\alpha$ is defined by the equation $g(1 \mathrm{AU})=1$. The scale distance $r_{0}$ is the heliocentric distance inside which the solar radiation leads to sublimation of water ices. At small solar distances $\left(r \ll r_{0}\right)$ it gives roughly an inverse-square force that describes the incident solar flux. At $r \gg r_{0}$ most of the solar radiation is thermally reradiated and $g(r)$ drops much faster with distance (see Fig. 1). This standard form of $g(r)$ is the most widely used in the literature for the short-period comets although there have been several different attempts to analyze the NG effects in cometary motion.

The standard $g(r)$ function can be used to introduce the general $g(r)$-like form, $g^{*}(r)$, where exponents $m, n, k$ and parameters $\alpha$ and $r_{0}$ are determined for each comet, individually. The general form of the NG acceleration is defined similarly to Eq. (2):

$F_{i}=A_{i}^{*} \cdot g^{*}(r), \quad A_{\mathrm{i}}^{*}=$ const. for $i=1,2,3$,

$g^{*}(r)=\alpha\left(r / r_{0}\right)^{-m}\left[1+\left(r / r_{0}\right)^{n}\right]^{-k}$ 


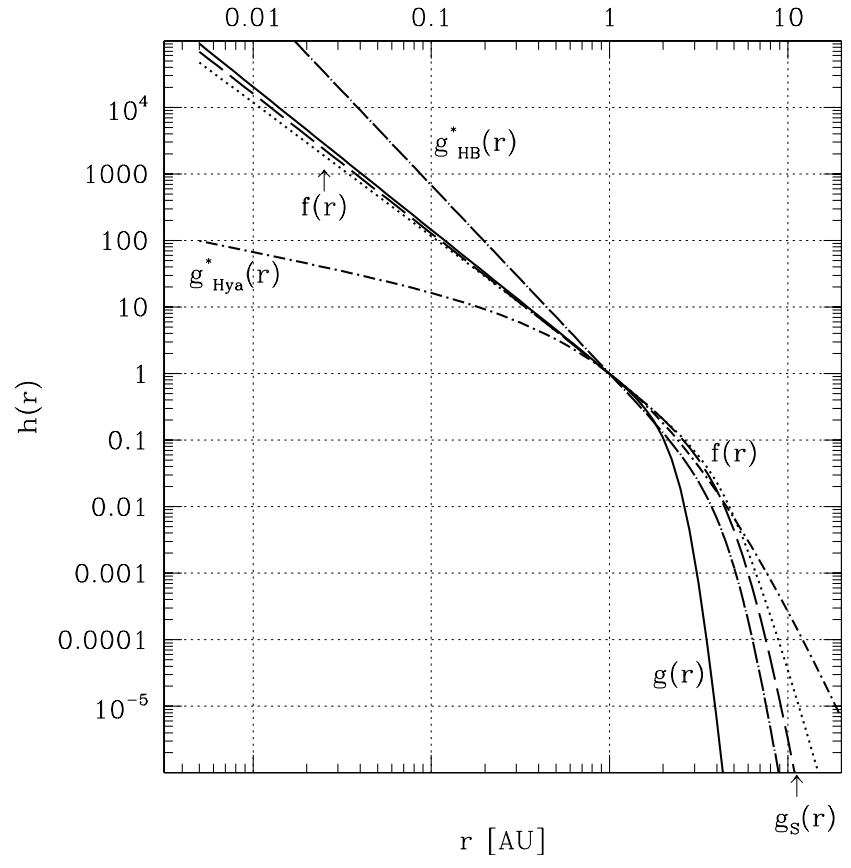

Fig. 1. Comparison of different forms of the NG function $h(r)$ (Eq. (5)) vs. heliocentric distance. Dotted-dashed curves marked as $g_{\mathrm{HB}}^{*}(r)$ and $g_{\mathrm{Hya}}^{*}(r)$ represent $g(r)$-like functions (Table 3) determined from the photometric observations for Comet C/1995 O1 Hale-Bopp and C/1996 B2 Hyakutake, respectively.

the present investigations three possibilities of the water sublimation model were considered:

A Water sublimation from the whole isothermal cometary nucleus. Such a model is sometimes called the "rapidly" rotating cometary nucleus. To determine the NG effects in the motion of a periodic comet the standard $g(r)$ function (Eqs. (1)-(2), Fig. 1) is commonly used in this model. In the case of the short-period comets, at least three consecutive apparitions are needed to determine precisely the independent NG parameters $A_{1}, A_{2}, A_{3}$ within an investigated time interval. This means that for one-apparition comets these effects cannot be determined: only about twenty long-period comets have the three NG constant parameters $A_{1}, A_{2}$ and $A_{3}$ determinable. Their values are given in Table 2. For each comet, the first line shows the values of $A_{1}$ and $A_{2}$ taken from the MW Catalogue (if they exist), in the second line the values of $A_{1}, A_{2}, A_{3}$ determined in the present investigation are given. For five comets (C/1996 B1, C/1959 Y1, C/1969 Y1, C/1005 O1, C/1998 P1) the models with an asymmetric function $g(r)$ were determinable. The respective values of $A_{1}, A_{2}, A_{3}$ and $\tau$ (time displacement of the maximum of $g(r)$ from the perihelion, see next section) are listed in the third line of Table 2 for these objects.

B Outgassing restricted to the subsolar point only. The appropriate constants (defined by Eq. (2)) were derived by Sekanina (1988), and are: $\alpha=0.02726, m=2.1, n=3.2$, $k=3.9$ and the scaling distance $r_{0}=5.6 \mathrm{AU}$ (by a factor of two greater than value for the "rapidly" rotating nucleus). The NG parameters determined in this model are indicated by $g_{\mathrm{S}}$ in Table 2 . The $r$-dependence of $g_{\mathrm{S}}(r)$ is shown by the
Table 3. Values of exponents $m, n, k$, the scale distance $r_{0}$, and coefficient $\alpha(g(r)$-like function, Eq. (2a)) estimated from observed water sublimation rates for C/1995 O1 Hale-Bopp and C/1996 B2 Hyakutake (Szutowicz et al. 2002a,b).

\begin{tabular}{ccc}
\hline \hline Coefficient & $\begin{array}{c}\text { Hale-Bopp } \\
\text { [function } g_{\mathrm{HB}}^{*}(r) \text { ] }\end{array}$ & $\begin{array}{c}\text { Hyakutake } \\
\text { [function } g_{\mathrm{Hya}}^{*}(r) \text { ] }\end{array}$ \\
\hline$\alpha$ & 0.008860 & 3.31564 \\
$m$ & 2.828 & 0.537 \\
$n$ & 3.76 & 1.0 \\
$k$ & 3.7 & 5.76 \\
$r_{0}(\mathrm{AU})$ & 5.3 & 2.808 \\
$Q_{m}$ (mol./s) & $8.9 \times 10^{30}$ & $1.9 \times 10^{29}$ \\
\hline
\end{tabular}

long-dashed curve in Fig. 1. This model assumes the existence of a discrete outgassing source (vent) on the surface of a rotating cometary nucleus.

C The values of exponents $m, n$ and $k$, the coefficient $\alpha$ and scale distance $r_{0}$ determined for each comet individually. Recently, Szutowicz et al. (2002a,b) modelled the NG motion of two well-known comets C/1995 O1 Hale-Bopp and C/1996 B2 Hyakutake. They fitted the observed water sublimation rates collected from the literature to the general form of the water sublimation rate as a function of the heliocentric distance:

$Q_{\mathrm{H}_{2} \mathrm{O}}(r)=Q_{m} \cdot \mathrm{g}^{*}(r)$,

where $Q_{m}$ is a constant parameter. The derived values of $\alpha$, $m, n, k$, and $r_{0}$ for Hale-Bopp and Hyakutake are given in Table 3. In the case of these two long-period comets the coefficients in Eq. (2a) could differ substantially from its canonical values derived by Marsden et al. (1973); especially the values of $m$ is significantly different from 2.15 , i.e. from a roughly inverse power law relation at small heliocentric distances. Similar investigations for the next three long-period comets are now in progress. However, for the remaining 14 comets the photometric observations are insufficient to determine the $g^{*}(r)$.

Therefore, the general form of $g^{*}(r)$ given by Eq. (2a) was also used here, in the solely orbital calculations for all the considered long-period comets. It turned out, however, that the rms determined from the positional data are only weakly sensitive to exponents $m, n$ and $k$. Only the scale distance $r_{0}$ could be determined for some comets. Three possibilities of $g^{*}(r)$ are considered here: $(a)$ the power law $(k=0) ;(b)$ the expressions differing from the standard function $g(r)$ only in the scale distance $r_{0}$; and $(c)-$ only the exponent $m$ is fitted. It appears that only the case (b) can provide better fits to the positional data. In our calculations we have found seven such $g(r)$-like solutions and in Table 2 they are denoted as $g(r)_{r_{0}}(\mathrm{C} / 1996 \mathrm{~B} 2$, C/1999 H1, C/1995 O1, C/1990 K1, 1999 J3, C/1998 P1, $\mathrm{C} / 1993 \mathrm{~A} 1)$. The next two $g(r)_{r_{0}}$ solutions are given for comets C/1991 T2 and C/1995 Y1 because they seem to be more physically realistic than those based on $g(r)$.

There are strong reasons to suppose that the sublimation from nearly parabolic comets may be controlled by $\mathrm{CO}$ or $\mathrm{N}_{2}$ 
molecules rather than the less volatile $\mathrm{H}_{2} \mathrm{O}$. Coming "fresh" from the Oort cloud these comets should exhibit a much higher content of the most volatile ices (e.g. $\mathrm{CO}, \mathrm{CO}_{2}$ ) that become substantially or completely depleted during their first perihelion passage.

Yabushita (1996) introduced a convenient form for the dependence of acceleration on the heliocentric distance based on sublimations of molecules of $\mathrm{CO}$ rather than $\mathrm{H}_{2} \mathrm{O}$ :

$F_{i}=A_{i}^{*} \cdot \mathrm{f}(r), \quad A_{i}^{*}=$ const. for $i=1,2,3$,

$f(r)=\frac{1.0006}{r^{2}} \times 10^{-0.07395(r-1)} \cdot\left(1+0.0006 r^{5}\right)^{-1}$,

where $r$ is in astronomical units. The original form of the $f(r)$-function given by Yabushita was renormalized here to give $f(r=1 \mathrm{AU})=1$ (see the dotted curve in Fig. 1).

To apply Eqs. (2), (2a) and (4) of NG accelerations to asymmetric cases with respect to perihelion we simply substitute $g\left(r^{\prime}\right), g^{*}\left(r^{\prime}\right)$ and $f\left(r^{\prime}\right)$ instead of $g(r), g^{*}(r), f(r)$, respectively, where $r^{\prime}=r(t-\tau)$; here $\tau$ represents the time displacement of the maximum of the relevant functions with respect to the perihelion.

Finally, for each comet the following forms of the NG acceleration were tested:

$F_{i}=A_{i}^{*} \cdot h(r), \quad A_{i}^{*}=$ const. for $i=1,2,3$,

$h(r)=\left\{\begin{array}{cc}g(r) & \text { standard Marsden function } \\ g(r(t-\tau)) & \text { asymmetric } g(r) \\ g_{\mathrm{S}}(r) & \text { subsolar outgassing } \\ g_{\mathrm{S}}(r(t-\tau)) & \text { asymmetric } g_{\mathrm{S}}(r) \\ g^{*}(r) & g(r)-\text { like function; Eq. (2a) } \\ g^{*}(r(t-\tau)) & \text { asymmetric } g^{*}(r) \\ f(r) & \text { CO subl.; Eq. (4) } \\ f(r(t-\tau)) & \text { asymmetric } f(r) .\end{array}\right.$

Since the asymmetric NG models introduce the additional parameter $\tau$, the task to determine the four NG parameters from the orbital motion of one-apparition comets becomes even more difficult.

Sets of models described by Eq. (5) are presented in Table 2. The NG parameters $A_{1}^{*}, A_{2}^{*}$ and $A_{3}^{*}$ resulting from the orbital fitting to observations for three different models including $g(r), g_{\mathrm{S}}(r)$ and $f(r)$ are given. For two comets $(\mathrm{C} / 1991 \mathrm{~T} 2$ and $\mathrm{C} / 1995 \mathrm{Y} 1)$ the models with standard $g(r)$ give $\left|A_{1}\right|<\left|A_{2}\right|$, therefore the best $g^{*}(r)$ solutions are presented. Asymmetric NG models were determinable only for five comets: C/1996 B2, C/1959 Y1, C/1969 Y1, C/1995 O1 and $\mathrm{C} / 1998 \mathrm{P} 1$.

For most of the investigated comets the NG parameters for the rotating spherical nucleus were also determined. In such a model $A_{1}^{*}, A_{2}^{*}$ and $A_{3}^{*}$ are now functions of time by relations: $A_{i}^{*}=A^{*} \cdot C_{i}(t), i=1,2,3$, where $C_{i}(t)$ are direction cosines for the NG force acting on the rotating cometary nucleus (Sitarski 1990). Now, three of the NG parameters are angular parameters: $\eta$ - the lag angle of the maximum outgassing behind subsolar meridian, $I$ - equatorial obliquity and $\phi-$ cometocentric solar longitude at perihelion. The values of $A, \eta, I$ and $\phi$, are presented in Table 4.

An assumption of a flattened nucleus represents the next step towards a more realistic cometary model. In this case, the forced precession of the spin axis could arise due to torque if a vector of the jet force does not pass through the center of the nonspherical nucleus. The precession rate is a function of the nucleus orientation, the lag angle, $\eta$, the modulus of the reactive force, $A$, the nucleus oblateness, $s$, and the precession factor, $f_{\mathrm{p}}$, which depends on the rotation period and nucleus size. In such a model the six parameters are derived: $A, \eta, I, \phi, s$ and $f_{\mathrm{p}}$ (Królikowska et al. 1998b). This procedure could be ambiguous for a one-apparition comet and it was possible for only three of 19 investigated objects: C/1993 A1 Mueller, C/1995 O1 Hale-Bopp and C/1996 B2 Hyakutake; the detailed descriptions of forced precession models for the latter two comets are given by Szutowicz et al. (2002a,b). In the case of C/1993 A1 the rotating nonspherical model was fully determinable only for the $f(r)$-function. For that comet the rms was reduced both for the constant NG parameters (Table 2) and for the forced precession model (Table 4). All these arguments indicate that the $\mathrm{CO}$ sublimation form described by $f(r)$ is strongly preferred. This result is also consistent with the small value of $1 / a_{\text {ori }}$ (Table 1 ) which implies that the comet C/1993 A1 Mueller is dynamically young.

For the next two comets (C/1999 J3 and C/1998 P1) the best fitted models for the rotating non-spherical nucleus are given. However, in both cases the values of $f_{\mathrm{p}}$ and $s$ are assumed rather than fitted, because it was impossible to determine all six parameters from the observational data. The case of C/1999 J3 is very special, because a forced precession model with the standard $g(r)$ (also with $f(r)$, and $g_{\mathrm{S}}(r)$ ) gives a negative $\eta$. Such a solution is physically unacceptable. This could suggest another $g^{*}(r)$-like function with a small value of the exponent $m$ (the similar form as derived for 1996 B2 Hyakutake). When $g_{\mathrm{Hya}}^{*}(r)$ was used, the forced precession model gave a positive $\eta$ (Table 4 ) but parameters $f_{\mathrm{p}}$ and $s$ could not be determined precisely. Clearly, the NG behaviour of the rotating nonspherical nucleus of C/1999 J3 Linear requires further analysis.

\section{Results and discussion}

The principal results and conclusions from the present study of the NG motion of nineteen long-period comets are the following.

1. New comets with detectable NG effects For six comets NG effects were detected in their orbital motion for the first time: C/1999 H1 Lee, C/1991 T2 ShoemakerLevy, C/1999 J3 Linear C/1975 T1 Mori-Sato-Fujikawa, C/1987 W2 Furuyama, C/1991 B1 Shoemaker-Levy.

2. Normal component $A_{3}^{*}$ For most of the investigated comets the normal component of the NG acceleration was determined for the first time. It turns out that only for five comets (C/1959 Y1, C/1995 Y1, C/1993 A1, C/1999 T1 and $\mathrm{C} / 1991 \mathrm{~B} 1$ ) could this component be neglected in comparison with the radial and transverse components since $\left|A_{3} / A_{2}\right|<0.1$. However, for two of them $(\mathrm{C} / 1999 \mathrm{~T} 1$ and $\mathrm{C} / 1991 \mathrm{~B} 1)$ the ratio $\left|A_{3}^{*} / A_{2}^{*}\right|$ grows when the Sekanina model is used, and for the next two comets (C/1959 Y1, C/1995 Y1) the normal component increases by a factor of 10 or more, while the transverse 
Table 5. Comparison between mean values of the NG parameters derived from models with constant $A_{1}, A_{2}, A_{3}$ and from models with a rotating spherical nucleus for the investigated sample of long-period (LP) comets and 20 short-period comets. Comets C/1991 T2 and C/1995 Y1 were excluded from the basic statistical analysis (17 LP comets) because another relations of $h(r)\left(g^{*}(r)\right.$ with $k=0$ i.e. the power law or $g(r)$-like function with $r_{0} \neq 2.808 \mathrm{AU}$ ) probably are more adequate for them (see Table 2). Two next two comets $(\mathrm{C} / 1998 \mathrm{P} 1$ and C/1993 A1) with the greatest NG parameters and $(1 / a)_{\text {ori }}<10^{-4}$ are excluded from the sample of $15 \mathrm{LP}$ comets. LP models of a rotating spherical nucleus are taken from Table 4.

I. Long period comets; models with constant $A_{1}, A_{2}$ and $A_{3}$

\begin{tabular}{cccccc} 
& \multicolumn{3}{c}{ 17 LP comets } & 19 LP comets & 15 LP comets \\
Models with: & $g(r)$ & $g_{\mathrm{S}}(r)$ & $f(r)$ & $g(r)_{\text {best } r_{0}}$ & $g(r)$ \\
$\left\langle A^{*}\right\rangle_{\text {weighted }} \times 10^{8} \mathrm{AU}$ day $^{-2}$ & $1.75 \pm 0.02$ & $1.59 \pm 0.02$ & $1.21 \pm 0.02$ & $1.89 \pm 0.02$ & $1.53 \pm 0.02$ \\
$\left\langle\left|A_{2}^{*} / A_{1}^{*}\right|\right\rangle_{\text {weighted }}$ & $0.065 \pm 0.003$ & $0.056 \pm 0.003$ & $0.054 \pm 0.003$ & $0.052 \pm 0.003$ & $0.081 \pm 0.004$ \\
$\left\langle F / F_{\odot}\right\rangle_{\text {weighted }} \times 10^{5}$ & $5.94 \pm 0.07$ & $5.71 \pm 0.07$ & $4.42 \pm 0.07$ & $5.80 \pm 0.07$ & $5.20 \pm 0.07$ \\
& & & & & \\
$\left\langle A^{*}\right\rangle \times 10^{8} \mathrm{AU}$ day $^{-2}$ & $6.43 \pm 0.13$ & $2.85 \pm 0.17$ & $2.40 \pm 0.07$ & $4.52 \pm 0.09$ & $4.07 \pm 0.15$ \\
$\left\langle\left|A_{2}^{*} / A_{1}^{*}\right|\right\rangle$ & $0.16 \pm 0.02$ & $0.20 \pm 0.05$ & $0.20 \pm 0.03$ & $0.23 \pm 0.02$ & $0.17 \pm 0.02$ \\
$\left\langle F / F_{\odot}\right\rangle \times 10^{5}$ & $21.8 \pm 0.4$ & $14.1 \pm 0.6$ & $11.6 \pm 0.2$ & $15.0 \pm 0.2$ & $13.8 \pm 0.4$ \\
\hline
\end{tabular}

II. Models of a rotating spherical nucleus

14 long-period comets

(spherical nucleus)

Models with:

$g(r)$

$g_{\mathrm{S}}(r)$

$f(r)$

$\left\langle A^{*}\right\rangle_{\text {weighted }} \times 10^{8} \mathrm{AU}_{\text {day }^{-2}}$

$\langle\eta\rangle_{\text {weighted }}$

$2.27 \pm 0.03$

$2.16 \pm 0.03$

$9.4 \pm 0.3$

$7.3 \pm 0.22$

$7.70 \pm 0.07$

$7.35 \pm 0.07$

$7.45 \pm 0.20$

$18.8 \pm 1.32$

$4.44 \pm 0.11$

$\left\langle A^{*}\right\rangle \times 10^{8} \mathrm{AU}$ day $^{-2}$

$\langle\eta\rangle$

$25.3 \pm 0.7$

$16^{\circ} .0 \pm 1.2$

$7.5 \pm 0.2$

$7.97 \pm 0.07$

$\left\langle F / F_{\odot}\right\rangle \times 10^{5}$

$15.1 \pm 0.4$

$4.05 \pm 0.10$

$16.3 \pm 1.1$

$2.35 \pm 0.02$

20 short-period comets

(non-spherical nucleus)

$15^{\circ} .1 \pm 1^{\circ} .2$

$1.66 \pm 0.14$

component decreases, if the asymmetric function $\mathrm{g}\left(r^{\prime}\right)$ is assumed.

The normal components $\left|A_{3}\right|$ are comparable to or larger than the transverse component $\left|A_{2}\right|$ for eight comets $(\mathrm{C} / 1996 \mathrm{~B} 2$ C/1999 H1, C/1993 Y1, C/1990 K1, C/1998 P1, C/1986 P1A, $\mathrm{C} / 1998 \mathrm{~T} 1, \mathrm{C} / 1987 \mathrm{~W} 2)$. In the extreme case of Comet $\mathrm{C} / 1993 \mathrm{Y} 1$, the value of $\left|A_{3}\right|$ is about five times larger than $\left|A_{2}\right|$. One should note, however, that in the present solution the amplitude of $A_{2}=-0.02 \times 10^{-8} \mathrm{AUday}^{-2}$ ) is much smaller than the value given in the MW Catalogue, where $A_{2}=+0.65 \times 10^{-8} \mathrm{AU} \mathrm{day}^{-2}$ and $A_{3} \equiv 0$.

3. Transverse component $A_{2}^{*}$ In the majority of cases the sign of the transverse component $A_{2}^{*}$ does not depend on the assumed symmetric model of $h(r)$ (see Table 2 ). For two comets (C/1990 K1 and C/1986 P1A) $A_{2}$ is positive, while $A_{2}^{*}$ derived for $g_{\mathrm{S}}(r)$ and $f(r)$ are negative.
Absolute values of $A_{2}$ span a very wide range and lie between $0.003(\mathrm{C} / 1993 \mathrm{Y} 1)$ and $2.9(\mathrm{C} / 1991 \mathrm{~T} 2)$ in the units of $10^{-8} \mathrm{AU}$ day $^{-2}$. Similar to the short period comets, positive and negative values of $A_{2}$ seem to be equally probable, and generally are an order of magnitude smaller than the radial component $A_{1}$. The mean values of the $\left|A_{2}^{*} / A_{1}^{*}\right|$ ratio are given in Part I of Table 5 .

4. Radial component $\boldsymbol{A}_{1}^{*}$ The radial term of the NG acceleration is always greater than the two remaining components. However, for only one comet in the sample (C/1986 P1A), the radial component distinctly dominates the remaining two: $\left|A_{1}\right| \gg\left|A_{2}\right|,\left|A_{3}\right|$. Thus, the simplest model with the only radial component seems to adequately describe the NG motion of 1986 P1A. Moreover, the errors of the parameters $A_{2}$ and $A_{3}$ are of the same order as the parameters themselves. 
The maximum value of the radial NG force which affected the cometary motion, $F_{\mathrm{NG} \text {,max }}^{*}$, could be calculated from the $A_{1}^{*}$ parameter according to relation:

$F_{\mathrm{NG}, \text { max }}^{*}=F_{1}^{*}(r=q)=A_{1}^{*} \cdot h(r=q)$.

Figure 3 shows the maximum values of the radial NG-forces as the function of the perihelion distance for all the investigated long-period comets. The $F_{\mathrm{NG} \text {,max }}^{*}$ values are in the range $\left(10^{-5}-10^{-3}\right) \times F_{\odot}(r)$, where solar gravitational acceleration satisfies the equation: $F_{\odot}(r)=0.59 \cdot r^{-2} \mathrm{~cm} \mathrm{~s}^{-2}$, and $r$ is in astronomical units (the dotted curves in Fig. 3 ).

\section{Time shift of the maximum of vaporization curve or/and} displacement of the photometric center There are two points which should be taken into account in the modelling of the NG effects:

(a) water vaporization curves peak a certain number of days, $\tau$, either before or after perihelion passage;

(b) the center of mass does not coincide with the photometric center of a comet's image.

It turns out, however, that the time shift $\tau$ is firmly determinable in the NG model with constants $A_{1}^{*}, A_{2}^{*}, A_{3}^{*}$ for only 5 of 19 long-period comets (C/1996 B2 Hyakutake, C/1959 Y1 Burnham, C/1969 Y1 Bennett, C/1995 O1 Hale-Bopp and C/1998 P1 Williams; see Table 2).

The problem of displacement of the photometric center, $\Delta r$, from the gravitational one is more complicated because the shape of a comet's image strongly depends on heliocentric distance; hence it is an a priori unknown function of $r$. Sitarski (1984) fitted the general form of $\Delta r=D \cdot r^{-k}$, where $k=1,2,3,4,5$, to the 37 positional observations of Comet C/1959 Y1 Burnham made by Roemer et al. (1966). The best orbit improvement was derived by him for exponents $k=3$ and $k=4$. He concluded that the relation $\Delta r=D \cdot r^{-3} \mathrm{e}^{-r^{2} / 2}$, similar to the form proposed by Marsden (1969) as Style I NG effects, represents the best model of displacement of the photometric center from the center of mass. Application of this method to the long-period comets provides ambiguous results. It turns out that only the observations of comet C/1959 Y1 Burnham indicate that such displacement could be real. In this case the NG solution with $\Delta r\left(\mathrm{rms}=1^{\prime \prime}\right.$. 12$)$ is the best one in comparison to the NG solution alone ( $\left.\mathrm{rms}=11^{\prime \prime} 46\right)$ as well as NG solution including $\tau$ (rms $=1$ ". 37 ) (Table 2). The derived values of parameters $A_{1}$ and $D$ are comparable to those obtained by Sitarski (1984). The present value of $A_{2}$ is significantly different because Sitarski assumed $A_{3}=0$, and in consequence, he obtained the value of $A_{2}$ nearly equal to zero. However, in this case, observations produced by only one team of observers (Roemer et al. 1966) have been used. When more observations of comet Burnham were taken into account this discrepancy is substantially reduced. Thus, the value of $D$ derived from the observations made by one observer (or one team of observers) could suffer from large errors and should not be used to determine the displacement of the photometric center. The appropriate choice between all reasonable models has a consequence on the shape of the original orbit. For the symmetric NG model the value of $1 / a_{\text {ori }}$ is negative (see Table 1 ) whereas for both the asymmetric model with $\tau$ and the symmetric model with assumed displacement of the photometric center the values of $1 / a_{\text {ori }}$ are positive and equal to $3 \times 10^{-6} \mathrm{AU}^{-1}$ and $62 \times 10^{-6} \mathrm{AU}^{-1}$, respectively. An asymmetric model of comet Burnham was also deduced by Matese \& Whitman (1993).

6. Mean values of NG accelerations For the investigated sample of long-period comets the mean value of a NG parameter $\left\langle A^{*}\right\rangle$, where $A^{*}=\sqrt{\left(A_{1}^{*}\right)^{2}+\left(A_{2}^{*}\right)^{2}+\left(A_{3}^{*}\right)^{2}}$, could also be determined for three assumed forms of $h(r): g(r), g_{\mathrm{S}}(r)$ and $f(r)$ (Table 5).

However, the range of $A$ (and $A^{*}$ ) values are broad (Fig. 2) and covers almost two orders of magnitude from 0.88 (Comet $\mathrm{C} / 1998 \mathrm{~T} 1$ ) to 32.2 (Comet $\mathrm{C} / 1998 \mathrm{P} 1$ ) in the units of $10^{-8} \mathrm{AU} \mathrm{day}^{-2}$. Also the errors of $A$ are spread over a wide range and are distributed between 0.03 and 1.7 (in the same units). In consequence, the arithmetic mean values of $A^{*}$ (Table 5) differ significantly from the weighted mean value of $A^{*}$. Thus, the mean values of $\left\langle A^{*}\right\rangle$ estimated from the three constant components $A_{1}^{*}, A_{2}^{*}$ and $A_{3}^{*}$ should be treated very carefully. Using $\left\langle A^{*}\right\rangle$ or $\left\langle A^{*}\right\rangle_{\text {weighted }}$ the mean value of NG acceleration at $1 \mathrm{AU}$ from the Sun, $F / F_{\odot}$, could be obtained, where $F_{\odot}=0.59 \mathrm{~cm} \mathrm{~s}^{-2}$ is the solar gravitational acceleration at $1 \mathrm{AU}$ (Table 5).

The largest absolute values of the $A^{*}$ parameter (or radial $A_{1}^{*}$ parameter) are likely associated with comets having a large perihelion distance, $q$, and/or having small reciprocals of the original semimajor axis, $1 / a_{\text {ori }}$ (Fig. 2). Therefore, it is justified to divide the whole sample in two subgroups with $q<1 \mathrm{AU}$ and $q>1 \mathrm{AU}$, respectively. The values of $A$ for the comets with q greater than $1 \mathrm{AU}$ are systematically greater and significantly more scattered than the corresponding distribution for the comets with $q$ smaller than 1 AU (upper panel of Fig. 2). The respective mean values of $A$ calculated for both samples are: $2.54 \pm 0.07(q<1)$ and $9.89 \pm 0.25(q>1)$ in units of $10^{-8} \mathrm{AU}_{\text {day }^{-2}}$. A similar result could be obtained by dividing the whole sample in two groups with $1 / a_{\text {ori }}<500 \times 10^{-6} \mathrm{AU}^{-1}$ and $1 / a_{\text {ori }}>500 \times 10^{-6} \mathrm{AU}^{-1}$ (middle panel of Fig. 2). Then, the mean values are $11.52 \pm 0.30$ and $2.87 \pm 0.10$, respectively. The correlation between both types of division is visualized in the bottom panel of Fig. 2. However, the investigated sample of long-period comets is too small to make a more detailed statistical analysis.

The mean values of $A^{*}$ for the model of the rotating spherical nucleus were also determined, using the sample of 14 comets from Table 4. It turns out that the mean values of $\left\langle A^{*}\right\rangle$ estimated from the models of a rotating cometary nucleus (Part II of Table 5) are similar to previous mean values.

It is interesting to compare these mean values with similar values estimated for short-period comets. Typically, observations of Jupiter-family comets cover several apparitions over several dozen years and for some comets even more than one century. In such cases the models based on constant NG parameters $A_{1}, A_{2}, A_{3}$ are fitted significantly less well to the whole interval of observations than models with a 


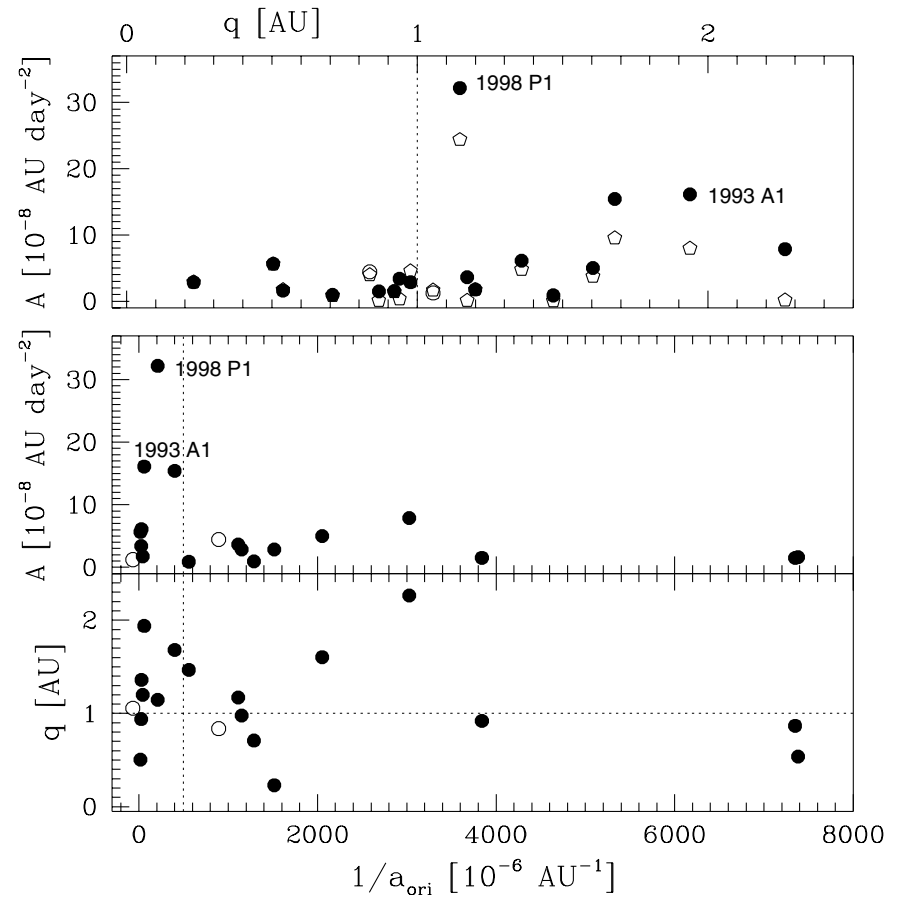

Fig. 2. The NG standard parameter $A=\sqrt{\left(A_{1}\right)^{2}+\left(A_{2}\right)^{2}+\left(A_{3}\right)^{2}}$ vs. the perihelion distance (upper panel) and reciprocals of original semimajor axis $1 / a_{\text {ori }}$ (middle) for the sample of 19 long-period comets. C/1991 T2 and C/1995 Y1 are shown by open circles; for these comets NG parameters $\mathrm{A}^{*}$ based on $g(r)$-like form are given (see Table 2). The $\mathrm{A}^{*}$ determined for the model with $g(r)_{r_{0} \neq 2.808 \mathrm{AU}}$ (see Table 2) are given by open pentagons in the upper panel. In the bottom panel the perihelion distances vs. $1 / a_{\text {ori }}$ for 19 comets are shown.

rotating nonspherical nucleus. It is illustrated by the following examples. I choose four Jupiter-family comets with different amplitudes of NG effects: 31P/Schwassmann-Wachmann 2, 46P/Wirtanen, 88P/Howell and 26P/Grigg-Skjellerup. Table 6 gives the NG solutions for models with constant $A_{1}, A_{2}, A_{3}$ and models with a rotating nonspherical nucleus, i.e. forced precession models. It is clearly visible that the forced precession models fit the observations with significantly better rms than models with constant NG parameters. Thus, the models with a rotating non-spherical nucleus are the most appropriate to calculate the mean value of $A$ from positional observations. Detailed studies of the NG effects that influence the motion of short-period comets have been undertaken by Sitarski and collaborators. They showed (Sitarski 1992, 1994; Królikowska \& Szutowicz 1999; Królikowska et al. 1998a,b, 2001; Szutowicz $1999,2000)$ that the forced precession model of the rotating cometary nucleus successfully describes the long-term motion of these comets. They obtained NG parameters for 20 shortperiod comets (some of these models have not been published yet). The individual values of $A$ have been found to range over 2 orders of magnitude between 0.02 (26P/GriggSkjellerup) and 1.4 (31P/Schwassmann-Wachmann 2) in the units of $10^{-8} \mathrm{AU}$ day $^{2}$. Table 5 gives the mean values of $A$, $\eta$ and $F / F_{\odot}$ for the sample of 20 short-period comets. These mean values (fifth column of Table 5) could be directly compared with the respective mean values obtained for long-period

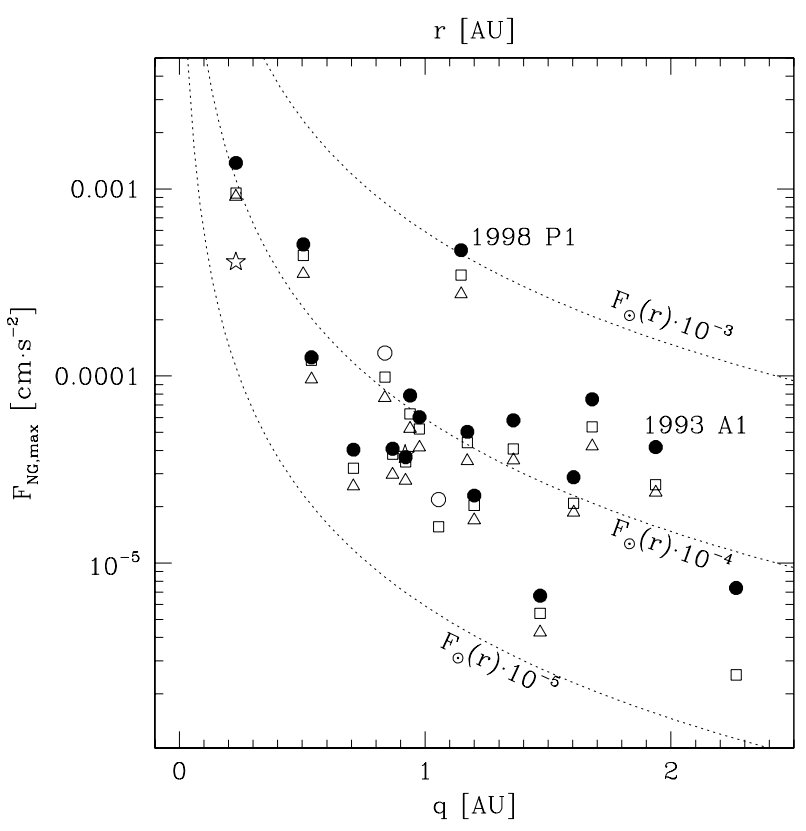

Fig. 3. Maximum of NG forces $F_{\mathrm{NG}, \max }=A^{*} \cdot h(r=q)$ (given in units of $\mathrm{cm} \mathrm{s}^{-2}$ ) vs. perihelion distance $q$ derived for the investigated longperiod comets. Circles, squares and triangles show maksimum values of NG-forces for the standard $g(r)$-function, $g_{\mathrm{S}}(r)$ and $f(r)$, respectively. The asterisks represent the $g_{\mathrm{Hya}}^{*}$ and $g_{\mathrm{HB}}^{*}$ models, for C/1996 B2 and $\mathrm{C} / 1995 \mathrm{O} 1$. The dotted curves represent the $10^{-3}, 10^{-4}, 10^{-5}$ of the solar gravitational acceleration $F_{\odot}(r)$, respectively.

comets (second column of Table 5). It turns out that the mean NG parameter $A$ estimated for the long-period comets is at least 10 times greater than the mean value of $A$ obtained for short-period comets. This is in very good agreement with the previous estimates based on the smaller sample of the longperiod comets. Marsden et al. (1973) determined the NG forces described by $A_{1}$, and $A_{2}$ from the sample of 23 short-period and 7 long-period comets. They obtained that the actual magnitude of the NG forces is typically about $10^{-5}$ times the solar attraction at $1 \mathrm{AU}$ for short-period comets and $(1-2) \times 10^{-4}$ of the solar attraction for the long-period comets.

7. Lag angle $\eta$ The range of $\eta$ - heat lag angles of the maximum outgassing behind the subsolar meridian - is $6^{\circ}-26^{\circ}$ for 12 long-period comets ( $86 \%$ of models of a rotating spherical nucleus). The remaining solutions yield angles $30^{\circ}-35^{\circ}$ (Comet C/1999 H1) and $43^{\circ}-59^{\circ}$ (Comet C/1991 T2). In the sample of 20 short-period comets all $\eta$ angles are below $30^{\circ}$. Table 5 shows that the mean values of $\eta$ calculated for the longperiod comets (Col. 2) somewhat exceed the mean values of $\eta$ derived for the short-period comets (Col. 5).

8. Which form of $h(r)$ is the most appropriate for the long-period comets? Effects of the assumed form of $h(r)$ on the NG parameters derived from the orbital calculations for the long-period comets have been investigated carefully in the present analysis. It is also important to examine which form of $h(r)$ is preferable for individual objects, i.e. which form of $h(r)$ gives the minimum rms in the orbital fitting procedure. Unfortunately, it is evident that the astrometric 
observations alone are not sensitive enough for such an analysis. Table 2 shows that a $g(r)$-law fit to the individual longperiod comet gives residuals that are essentially the same as those obtained from the $g(r)$-like law $\left(g_{\mathrm{S}}, g^{*}\right)$ and those derived from the $f(r)$-relation. Nevertheless, I would like to point to some tendencies which seem to be real.

As was discussed in Sect. 2 seven comets from the sample (C/1959 Y1, C/1990 K1, C/1995 Y1, C/1998 P1, C/1986 P1A, $\mathrm{C} / 1996 \mathrm{E} 1$, and C/1993 A1) seem to be dynamically new. For three of them the decrease of rms is visible when the $f(r)$-law rather than the $g(r)$-law is used. For Comet C/1998 P1 Williams the rms drops from 1'.10 to 1'.02, for C/1993 A1 Mueller - from 1".'14 to 1".11, and for C/1990 K1 Levy - from $1^{\prime \prime} 21$ to 1 '. 19 . For the remaining 12 comets only Comet C/1996 B2 Hyakutake exhibits a similar decrease of rms from $11^{\prime \prime} 08\left(g(r)\right.$-law) to $11^{\prime \prime} 05(f(r)$-law $)$. In the case of these three dynamically young comets the $g_{\mathrm{S}}(r)$-law or $g(r)$-like law with $r_{0} \neq 2.808$ AU give the same rms as $f(r)$, which further confuses the issue. However, the extremely large values of $A_{1}$ for C/1998 P1 Williams and C/1993 A1 Mueller are unrealistic in terms of the water sublimation (Whipple 1978; Yabushita 1991). This supports the idea that more volatile molecules than water play an important role in the orbital departure from the gravitational path for these two comets. Furthermore, the detailed investigations of C/1995 O1 HaleBopp and C/1996 B2 Hyakutake show that the $g(r)$-like forms derived from the photometric data are very different. This could suggest a large variety of NG behaviours in the population of the long-period comets.

The second interesting result is connected with the scale distance $r_{0}$. Marsden et al. (1973) showed that the value of the scale distance $r_{0}$ related to the Bond albedo, $A_{\text {vis }}$, and infrared albedo $A_{\mathrm{IR}}$, is equal to the standard $r_{0}=2.808 \mathrm{AU}$ whenever $A_{\mathrm{vis}} \simeq A_{\mathrm{IR}}$. Moreover, the $r_{0}$ differs significantly from $2.8 \mathrm{AU}$, while exponents $n, m$ and $k$ vary only slightly with the albedos. To investigate this question, I have tested the family of $g(r)$-like functions with the same standard values of exponents $n, m$ and $k$ and different values of $r_{0}: 1.5 \mathrm{AU}$ $\left(A_{\mathrm{vis}}>0.7\right), 2.0 \mathrm{AU}\left(A_{\mathrm{vis}}>0.5\right), 4 \mathrm{AU}\left(A_{\mathrm{IR}}>0.46\right), 6 \mathrm{AU}$ $\left(A_{\mathrm{IR}}>0.74\right), 8 \mathrm{AU}\left(A_{\mathrm{IR}}>0.83\right)$. In two cases where the standard $g(r)$ fitted to observations gave $\left|A_{2}\right|>\left|A_{1}\right|$, variation of $r_{0}$ allowed us to obtain reasonable values of the NG parameters: for C/1991 T2 with $r_{0} \geq 4 \mathrm{AU}$ and for C/1995 Y1 with $r_{0} \leq 1.6 \mathrm{AU}$ (Table 2). For the next seven comets the better fit to the positional observations is derived for other values of $r_{0}$ than the standard $r_{0}=2.808 \mathrm{AU}$. For three of those comets (C/1999 H1, C/1995 O1 Hale-Bopp and C/1999 J3) the smallest rms was obtained for $r_{0}=1.5 \mathrm{AU}$, for the remaining four (C/1996 B2, C/1990 K1, C/1998 P1, C/1993 A1) the best fit was found for the large value of $r_{0} \geq 4 \mathrm{AU}$. However, as was discussed above, for three objects from the last group (C/1990 K1, C/1998 P1, C/1993 A1) the $f(r)$-function also give a smaller rms than the standard $g(r)$-form.

\section{Future perspectives}

Present investigations show that astrometric observations alone are not sensitive enough to judge which form of $h(r)$ is appropriate in the analysis of the NG effects for individual long-period comets. Therefore, photometric observations are needed to solve this problem. The NG motion of two well-known comets, C/1995 O1 Hale-Bopp and C/1996 B2 Hyakutake, were recently modelled by Szutowicz et al. (2002a,b). They fitted the observed water sublimation rates and the observed light curve collected from the literature to the general $g(r)$-like form of the water sublimation rate. Since such analysis requires long series of spectroscopic (and/or photometric) observations it could be applied only to a few long-period comets. The $g(r)$-like functions obtained this way were then applied to determine the NG parameters. It appeared that in the case of comets Hale-Bopp and Hyakutake the coefficients in the $g(r)$-like law differ significantly (Fig. 1) from theirs canonical values derived by Marsden et al. (1973) (compare Table 3 and Eq. (2)). Similar investigations for the next three long-period comets are in progress.

We also intend to analyze the dynamical evolution of the long-period comets with respect to their NG effects. Some elements of these investigations limited only to NG dynamical evolution described by the $g(r)$-law are presented by Szutowicz et al. (2002a) and Królikowska (2002).

Acknowledgements. The author thanks Professors Grzegorz Sitarski and Andrzej M. Sołtan and Dr. Sławomira Szutowicz for valuable discussions. This work was supported by the Polish Commitee for Scientific Research (the KBN grant 2.P03D.002.09).

\section{References}

Bielicki, M., \& Sitarski, G. 1991, Acta Astron., 41, 309

van Biesbroeck, G., \& Marsden, B. G. 1963, AJ, 68, 566

Bolatto, A. D., Fernandez, J. A., \& Carballo, G. F. 1995, Planet. Space Sci., 43, 709

Delsemme, A. H., \& Miller, D. C. 1971, Planet. Space Sci., 19, 1229

Królikowska, M. 2001, A\&A, 376, 316, Paper I

Królikowska, M. 2002, Proceedings of Asteroids, Comets, Meteors ACM 2002, ESA SP-500, 629

Królikowska, M., \& Sitarski, G. 1996, A\&A, 310, 992

Królikowska, M., \& Szutowicz, S. 1999, A\&A, 343, 997

Królikowska, M., Sitarski, G., \& Szutowicz, S. 1998a, Acta Astron., 48,91

Królikowska, M., Sitarski, G., \& Szutowicz, S. 1998b, A\&A, 335, 757

Marsden, B. G. 1969, AJ, 74, 720

Marsden, B. G. 1970, AJ, 75, 75

Marsden, B. G., \& Sekanina, Z. 1973, AJ, 78, 1118

Marsden, B. G., Williams, G. V. 2003, Catalogue of Cometary Orbits, Fifteenth ed.

Marsden, B. G., Sekanina, Z., \& Yeomans, D. K. 1973, AJ, 78, 211

Marsden, B. G., Sekanina, Z., \& Everhart, E. 1978, AJ, 83, 64

Matese, J. J., \& Whitman, P. G. 1993, in Asteroids, Comets, Meteors 1993, 207

Matese, J. J., Whitman, P. G., \& Whitmire, D. P. 1992, in Asteroids, Comets, Meteors 1991, 399

Roemer, E., Thomas, M., \& Lloyd, R. E. 1966, AJ, 71, 591

Sekanina, Z. 1968, BAICz 19, 210

Sekanina, Z. 1988, AJ, 95, 911

Sitarski, G. 1984, Acta Astron., 34, 269

Sitarski, G. 1989, Acta Astron., 39, 345 
Sitarski, G. 1990, Acta Astron., 40, 405

Sitarski, G. 1992, Acta Astron., 42, 59

Sitarski, G. 1994, Acta Astron., 44, 91

Sitarski, G. 1998, Acta Astron., 48, 547

Sitarski, G. 2002, Acta Astron., 52, 471

Sitarski, G. 2003, private communication

Szutowicz, S. 2000, A\&A, 363, 323

Szutowicz, S., Królikowska, M., \& Sitarski, G. 2002a, Earth, Moon, and Planets, 90, 119
Szutowicz, S., Królikowska, M., \& Sitarski, G. 2002b, Proceedings of Asteroids, Comets, Meteors ACM 2002, ESA SP-500, 633

Weissman, P. R. 1979, AJ, 84, 580

Weissman, P. R. 1985, Space Sci. Rev., 41, 299

Whipple, F. L. 1977, in Comets, Asteroids, Meteorites ed. A. H. Delsemme, The University of Toledo, 25 Yabushita, S. 1979, MNRAS, 187, 445

Yabushita, S. 1991, Earth, Moon and Planets, 52, 87

Yabushita, S. 1996, MNRAS, 283, 347 


\section{Online Material}


M. Królikowska: Long-period comets with non-gravitational effects, Online Material $p 2$

Table 1. General characteristics of the observational material. The succesive columns signify: comet designation and name (Col. 1) perihelion time, $T$, and perihelion distance, $q$ (2), observational arc and number of all observations (3), rms for the pure gravitational orbital fitting to pre-periherlion (4), post-perihelion (5) and all (6) observations, rms for the NG orbital fitting to all observations (7), original (8) and future (9) reciprocals of semimajor axes in units of $10^{-6} \mathrm{AU}^{-1}$.

\begin{tabular}{|c|c|c|c|c|c|c|c|c|}
\hline \multirow[t]{2}{*}{$\begin{array}{c}\text { Comet } \\
\text { name }\end{array}$} & \multirow{2}{*}{$\begin{array}{c}\text { Perihelion } \\
\text { time } T \text { [Date] } \\
q[\mathrm{AU}]\end{array}$} & \multirow{2}{*}{$\begin{array}{c}\text { Observational } \\
\text { material } \\
\text { (Number of obs.) }\end{array}$} & \multicolumn{3}{|c|}{$\begin{array}{r}\mathrm{rms} \\
\text { Pure grav. case }\end{array}$} & \multirow{2}{*}{$\begin{array}{c}\text { NG case } \\
\text { all }\end{array}$} & \multirow{2}{*}{\multicolumn{2}{|c|}{$\begin{array}{c}(1 / a)_{\text {ori }} \quad(1 / a)_{\text {fu }} \\
\text { NG } \\
\text { (pure grav. ) }\end{array}$}} \\
\hline & & & $\begin{array}{l}\text { pre- } \\
\text { perih. }\end{array}$ & $\begin{array}{l}\text { post- } \\
\text { perih. }\end{array}$ & all & & & \\
\hline $\begin{array}{c}1996 \text { B2 } \\
\text { Hyakutake }\end{array}$ & $\begin{array}{c}19960501 \\
0.230\end{array}$ & $\begin{array}{c}19960101-19961102 \\
(1026)\end{array}$ & $\begin{array}{l}1 . .02 \\
(971)\end{array}$ & $\begin{array}{l}1 . \prime 19 \\
(55)\end{array}$ & 1." 66 & $1{ }^{\prime \prime} 08$ & $\begin{array}{c}1515 \\
(1400)\end{array}$ & $\begin{array}{c}554 \\
(540)\end{array}$ \\
\hline $\begin{array}{l}1959 \text { Y1 } \\
\text { Burnham }\end{array}$ & $\begin{array}{c}19600321 \\
0.504\end{array}$ & $\begin{array}{c}19600104-19600617 \\
(88)\end{array}$ & $\begin{array}{l}1.229 \\
(34)\end{array}$ & $\begin{array}{l}2, \cdot 14 \\
(54)\end{array}$ & $3 .^{\prime \prime} 00$ & 1.'64 & $\begin{array}{l}-202 \\
(-139)\end{array}$ & $\begin{array}{l}-319 \\
(-582)\end{array}$ \\
\hline $\begin{array}{l}1969 \mathrm{Y} 1 \\
\text { Bennett }\end{array}$ & $\begin{array}{c}19700320 \\
0.538\end{array}$ & $\begin{array}{c}19691230-19701027 \\
(548)\end{array}$ & $\begin{array}{l}0 ! \\
(159)\end{array}$ & $\begin{array}{l}1,68 \\
(389)\end{array}$ & $2 . \prime 00$ & $1{ }^{\prime \prime} 53$ & $\begin{array}{c}7381 \\
(7317)\end{array}$ & $\begin{array}{l}7126 \\
(7126)\end{array}$ \\
\hline $\begin{array}{l}1999 \mathrm{H} 1 \\
\text { Lee }\end{array}$ & $\begin{array}{c}19990711 \\
0.708\end{array}$ & $\begin{array}{c}19990416-20000108 \\
(1032)\end{array}$ & $\begin{array}{l}0 ! \\
(352)\end{array}$ & $\begin{array}{l}0.69 \\
(680)\end{array}$ & 0.98 & 0.69 & $\begin{array}{c}1288 \\
(1313)\end{array}$ & $\begin{array}{c}397 \\
(365)\end{array}$ \\
\hline $\begin{array}{c}1991 \mathrm{~T} 2 \\
\text { Shoemaker-Levy }\end{array}$ & $\begin{array}{c}19920724 \\
0.836\end{array}$ & $\begin{array}{c}19911006-19920821 \\
(212)\end{array}$ & $\begin{array}{l}1,221 \\
(209)\end{array}$ & $\begin{array}{l}- \\
\text { (3) }\end{array}$ & $1{ }^{\prime \prime} 21$ & 1.. 14 & $\begin{array}{c}894 \\
(941)\end{array}$ & $\begin{array}{l}1019 \\
(434)\end{array}$ \\
\hline $\begin{array}{c}1993 \text { Y1 } \\
\text { McNaught-Russell }\end{array}$ & $\begin{array}{c}19940331 \\
0.868\end{array}$ & $\begin{array}{c}19931217-19940908 \\
(397)\end{array}$ & $\begin{array}{l}0 . \\
(146)\end{array}$ & $\begin{array}{l}1 . \prime 12 \\
(251)\end{array}$ & 1.'23 & $1 . .00$ & $\begin{array}{c}7349 \\
(7171)\end{array}$ & $\begin{array}{c}8442 \\
(8261)\end{array}$ \\
\hline $\begin{array}{c}1995 \text { O1 } \\
\text { Hale-Bopp }\end{array}$ & $\begin{array}{c}19970401 \\
0.918\end{array}$ & $\begin{array}{c}19930427-20011217 \\
(3550)\end{array}$ & $\begin{array}{l}1 . \prime 06 \\
(2580)\end{array}$ & $\begin{array}{l}1, .06 \\
(970)\end{array}$ & 1.229 & $1{ }^{\prime \prime} 07$ & $\begin{array}{c}3841 \\
(3797)\end{array}$ & $\begin{array}{c}5588 \\
(5566)\end{array}$ \\
\hline $\begin{array}{l}1990 \mathrm{~K} 1 \\
\text { Levy }\end{array}$ & $\begin{array}{c}19901025 \\
0.939\end{array}$ & $\begin{array}{c}19900521-19920401 \\
(678)\end{array}$ & $\begin{array}{l}1 . \prime 18 \\
(590)\end{array}$ & $\begin{array}{l}1,22 \\
(88)\end{array}$ & $1{ }^{\prime \prime} 94$ & $1^{\prime \prime} 21$ & $\begin{array}{c}26 \\
(112)\end{array}$ & $\begin{array}{l}-790 \\
(-849)\end{array}$ \\
\hline $\begin{array}{c}1999 \mathrm{~J} 3 \\
\text { Linear }\end{array}$ & $\begin{array}{c}19990920 \\
0.977\end{array}$ & $\begin{array}{c}19990512-20000228 \\
(196)\end{array}$ & $\begin{array}{l}0 . ' 66 \\
(91)\end{array}$ & $\begin{array}{l}1, \prime 12 \\
(105)\end{array}$ & $1{ }^{\prime \prime} 25$ & $0{ }^{\prime \prime} 83$ & $\begin{array}{c}1150 \\
(1084)\end{array}$ & $\begin{array}{c}963 \\
(769)\end{array}$ \\
\hline $\begin{array}{c}1995 \text { Y1 } \\
\text { Hyakutake }\end{array}$ & $\begin{array}{c}19960224 \\
1.055\end{array}$ & $\begin{array}{c}19951226-19960921 \\
(262)\end{array}$ & $\begin{array}{l}0 ! \\
(185)\end{array}$ & $\begin{array}{l}0.72 \\
(77)\end{array}$ & $0 ! 96$ & $0 .{ }^{\prime} 80$ & $\begin{array}{l}-67 \\
(-4)\end{array}$ & $\begin{array}{c}649 \\
(1119)\end{array}$ \\
\hline $\begin{array}{c}1998 \text { P1 } \\
\text { Williams }\end{array}$ & $\begin{array}{c}19981017 \\
1.147\end{array}$ & $\begin{array}{c}19980811-19990515 \\
(461)\end{array}$ & $\begin{array}{l}0 ! 58 \\
(133)\end{array}$ & $\begin{array}{l}1,73 \\
(328)\end{array}$ & 4..'58 & 1." 10 & $\begin{array}{c}211 \\
(-125)\end{array}$ & $\begin{array}{c}1320 \\
(1119)\end{array}$ \\
\hline $\begin{array}{c}1999 \text { T1 } \\
\text { McNaught-Hartley }\end{array}$ & $\begin{array}{c}20001213 \\
1.172\end{array}$ & $\begin{array}{c}19991007-20011202 \\
(481)\end{array}$ & $\begin{array}{l}0 . \\
(260)\end{array}$ & $\begin{array}{l}0.45 \\
(442)\end{array}$ & $1{ }^{\prime \prime} 27$ & 0.76 & $\begin{array}{l}1111 \\
(1111)\end{array}$ & $\begin{array}{l}141 \\
(34)\end{array}$ \\
\hline $\begin{array}{l}\text { 1986 P1A } \\
\text { Wilson }\end{array}$ & $\begin{array}{c}19870421 \\
1.200\end{array}$ & $\begin{array}{c}19860805-19890411 \\
(688)\end{array}$ & $\begin{array}{l}1.331 \\
(507)\end{array}$ & $\begin{array}{l}1,32 \\
(181)\end{array}$ & $1{ }^{\prime \prime} 62$ & $1^{\prime \prime} 31$ & $\begin{array}{c}42 \\
(-0.4)\end{array}$ & $\begin{array}{c}768 \\
(725)\end{array}$ \\
\hline $\begin{array}{l}1996 \text { E1 } \\
\text { NEAT }\end{array}$ & $\begin{array}{c}19960727 \\
1.359\end{array}$ & $\begin{array}{c}19960315-19961012 \\
(249)\end{array}$ & $\begin{array}{l}0 ! \\
(177)\end{array}$ & $\begin{array}{r}0.75 \\
(72)\end{array}$ & $1{ }^{\prime \prime} 01$ & 0.77 & $\begin{array}{c}28 \\
(-43)\end{array}$ & $\begin{array}{c}34 \\
(36)\end{array}$ \\
\hline $\begin{array}{l}1998 \mathrm{~T} 1 \\
\text { Linear }\end{array}$ & $\begin{array}{c}19990625 \\
1.468\end{array}$ & $\begin{array}{c}19981002-20000508 \\
(466)\end{array}$ & $\begin{array}{l}0.59 \\
(263)\end{array}$ & $\begin{array}{l}0.77 \\
(203)\end{array}$ & 1." 43 & 0.70 & $\begin{array}{c}560 \\
(559)\end{array}$ & $\begin{array}{c}1481 \\
(1458)\end{array}$ \\
\hline $\begin{array}{c}1975 \text { T1 } \\
\text { Mori-Sato-Fujikawa }\end{array}$ & $\begin{array}{c}19751225 \\
1.604\end{array}$ & $\begin{array}{c}19751006-19761024 \\
(197)\end{array}$ & $\begin{array}{l}1.335 \\
(139)\end{array}$ & $\begin{array}{l}1,21 \\
(58)\end{array}$ & 1.' 48 & 1.. 31 & $\begin{array}{c}2052 \\
(1958)\end{array}$ & $\begin{array}{c}1572 \\
(1540)\end{array}$ \\
\hline $\begin{array}{c}1987 \text { W2 } \\
\text { Furuyama }\end{array}$ & $\begin{array}{c}19880303 \\
1.680\end{array}$ & $\begin{array}{c}19871121-19880812 \\
(154)\end{array}$ & $\begin{array}{l}0 ! 93 \\
(144)\end{array}$ & $\begin{array}{l}1,56 \\
(10)\end{array}$ & $1{ }^{\prime \prime} 18$ & $1{ }^{\prime \prime} 06$ & $\begin{array}{c}401 \\
(266)\end{array}$ & $\begin{array}{l}50 \\
(7)\end{array}$ \\
\hline $\begin{array}{l}1993 \text { A1 } \\
\text { Mueller }\end{array}$ & $\begin{array}{c}19940113 \\
1.938\end{array}$ & $\begin{array}{c}19921126-19940817 \\
(746)\end{array}$ & $\begin{array}{l}1.33 \\
(671)\end{array}$ & $\begin{array}{l}0.66 \\
(75)\end{array}$ & $2 . .79$ & $1{ }^{\prime \prime} 14$ & $\begin{array}{c}58 \\
(-19)\end{array}$ & $\begin{array}{l}-409 \\
(-539)\end{array}$ \\
\hline $\begin{array}{c}1991 \text { B1 } \\
\text { Shoemaker-Levy }\end{array}$ & $\begin{array}{c}19911231 \\
2.264 \\
\end{array}$ & $\begin{array}{c}19910113-19930815 \\
(429) \\
\end{array}$ & $\begin{array}{l}0.96 \\
(123) \\
\end{array}$ & $\begin{array}{l}0.92 \\
(306) \\
\end{array}$ & $1{ }^{\prime \prime} 06$ & 0.93 & $\begin{array}{r}3027 \\
(3019) \\
\end{array}$ & $\begin{array}{r}3100 \\
(3083) \\
\end{array}$ \\
\hline
\end{tabular}


M. Królikowska: Long-period comets with non-gravitational effects, Online Material p 3

Table 2. NG parameters derived from positional observations for assumed function $h(r)$ (Eq. (5)) represented by: standard function $g(r)$, $g^{*}(r)$ - modified function $g(r), g_{\mathrm{S}}(r)$ - Sekanina's function, $f(r)$ - Yabushita's function describing CO sublimation, respectively. NG parameters $A_{1}^{*}, A_{2}^{*}, A_{3}^{*}$ are given in units of $10^{-8} \mathrm{AU}$ day ${ }^{-2}$; these parameters are the standard $A_{1}, A_{2}$ and $A_{3}$ for the NG models including the standard $g(r) ; \tau$ is given in days. First line for each individual comet shows the NG parameters $A_{1}$ and $A_{2}$ taken from MW Catalogue or Minor Planet Circulars (if there are). The $g^{*}(r)$ functions for C/1995 O1 Hale-Bopp $\left(g_{\mathrm{HB}}^{*}(r)\right)$ and C/1996 B2 Hyakutake $\left(g_{\mathrm{Hya}}^{*}(r)\right)$ are described in Table 3. For comet $\mathrm{C} / 1959$ Y1 Burnham the models with displacement, $D$, of photometric centre from the gravitational one are also presented; the values of this displacement (in paranthesis) are given in units of $10^{-5} \mathrm{AU}$. Models with subscript ${ }^{R}$ indicate solutions based on 37 observations of Comet Burnham made by Roemer et al. (1966).

\begin{tabular}{|c|c|c|c|c|c|c|}
\hline Name & $h(r)$ & $A_{1}^{*}$ & $A_{2}^{*}$ & $A_{3}^{*}$ & $\begin{array}{c}\tau \\
{[D]}\end{array}$ & $\mathrm{rms}$ \\
\hline \multirow[t]{10}{*}{1996 B2 } & $g(r)_{\mathrm{Mar}}$ & +2.76 & +0.0716 & & & \\
\hline & $g(r)$ & $+2.7699 \pm .1793$ & $+0.1396 \pm .0090$ & $+0.6602 \pm .0678$ & & 1.' 08 \\
\hline & $g(r)$ & $+4.0727 \pm .0776$ & $-0.3590 \pm .0366$ & $-0.9748 \pm .0500$ & $1.834 \pm .095$ & 0.98 \\
\hline & $g_{\mathrm{S}}(r)$ & $+2.0592 \pm .0532$ & $+0.1322 \pm .0087$ & $-0.5466 \pm .0608$ & & $1^{\prime \prime} 06$ \\
\hline & $g_{\mathrm{S}}(r)$ & $+3.6962 \pm .0705$ & $-0.3319 \pm .0358$ & $-0.7876 \pm .0452$ & $1.862 \pm .103$ & 0.97 \\
\hline & $f(r)$ & $+2.0630 \pm .1793$ & $+0.1078 \pm .0070$ & $-0.4160 \pm .0483$ & & 1.. 05 \\
\hline & $f(r)$ & $+2.9400 \pm .0559$ & $-0.2651 \pm .0288$ & $-0.6080 \pm .0366$ & $1.896 \pm .105$ & 0.97 \\
\hline & $g_{\mathrm{Hya}}^{*}(r)$ & $+0.6216 \pm .0103$ & $+0.0704 \pm .0032$ & $-0.0034 \pm .0129$ & & 0.97 \\
\hline & $g_{\mathrm{Hya}}^{*}(r)$ & $+0.8538 \pm .1759$ & $-0.0686 \pm .0117$ & $-0.0330 \pm .0111$ & $2.936 \pm .174$ & 0.92 \\
\hline & $g(r)_{r_{0}=4 \mathrm{AU}}$ & $+2.7889 \pm .0585$ & $+0.1383 \pm .0091$ & $-0.6442 \pm .0660$ & & 1.. 07 \\
\hline \multirow[t]{11}{*}{1959 Y1 } & $g(r)_{\mathrm{Mar}} R$ & +5.9 & -1.4 & & & \\
\hline & $g(r)$ & $+5.3028 \pm .4385$ & $-0.6879 \pm .3349$ & $+0.1774 \pm .1238$ & & 1." 64 \\
\hline & $g(r)$ & $+5.1843 \pm .4191$ & $+0.2103 \pm .3950$ & $+0.2932 \pm .1218$ & {$[.5606 \pm .1453]$} & 1.. 56 \\
\hline & $g(r)$ & $+9.661 \pm 1.314$ & $+1.3142 \pm .5776$ & $+0.7462 \pm .2012$ & $-9.37 \pm 1.51$ & 1.' 59 \\
\hline & $g_{\mathrm{S}}(r)$ & $+4.8533 \pm .3975$ & $-0.6092 \pm .3170$ & $+0.1449 \pm .1183$ & & 1.' 64 \\
\hline & $g_{\mathrm{S}}(r)$ & $+8.678 \pm 1.111$ & $+1.2406 \pm .5467$ & $+0.6558 \pm .1882$ & $-9.61 \pm 1.62$ & $1^{\prime \prime} .59$ \\
\hline & $f(r)$ & $+3.8495 \pm .3156$ & $-0.4864 \pm .2511$ & $+0.1147 \pm .0936$ & & 1.' 64 \\
\hline & $f(r)$ & $+6.8991 \pm .8843$ & $+0.9821 \pm .4330$ & $+0.5221 \pm .1492$ & $-9.60 \pm 1.61$ & $1^{\prime \prime} .59$ \\
\hline & $g(r)^{R}$ & $+5.5372 \pm .4688$ & $-1.1247 \pm .4190$ & $+0.0400 \pm .1503$ & & 1." 46 \\
\hline & $g(r)^{R}$ & $+5.3533 \pm .3590$ & $+0.5632 \pm .4027$ & $+0.2872 \pm .1204$ & {$[.8467 \pm .1229]$} & $1^{\prime \prime} 12$ \\
\hline & $g(r)^{R}$ & $+10.688 \pm 1.273$ & $+1.6942 \pm .6389$ & $+0.8080 \pm .2791$ & $-10.54 \pm 1.36$ & 1.. 37 \\
\hline \multirow[t]{7}{*}{$1969 \mathrm{Y} 1$} & $g(r)_{\mathrm{Mar}}$ & +1.9 & +0.1 & & & \\
\hline & $g(r)$ & $+1.6024 \pm .1793$ & $+0.1634 \pm .1462$ & $-0.0988 \pm .2059$ & & 1.. 53 \\
\hline & $g(r)$ & $+1.6668 \pm .1426$ & $+0.5054 \pm .1426$ & $+0.3811 \pm .1950$ & $+6.23 \pm 1.66$ & $1^{\prime \prime} .53$ \\
\hline & $g_{\mathrm{S}}(r)$ & $+1.6070 \pm .1668$ & $+0.2178 \pm .1210$ & $+0.0253 \pm .1783$ & & $1^{\prime \prime} .53$ \\
\hline & $g_{\mathrm{S}}(r)$ & $+1.6074 \pm .1339$ & $+0.4563 \pm .1194$ & $+0.3607 \pm .1680$ & $+5.96 \pm 1.69$ & $1^{\prime \prime} .53$ \\
\hline & $f(r)$ & $+1.2698 \pm .1321$ & $+0.1709 \pm .0959$ & $+0.0169 \pm .1413$ & & $1^{\prime \prime} .53$ \\
\hline & $f(r)$ & $+1.2700 \pm .1057$ & $+0.3598 \pm .0944$ & $+0.2823 \pm .1326$ & $+5.94 \pm 1.69$ & 1.' 53 \\
\hline \multirow[t]{5}{*}{1999 H1 } & $g(r)_{\mathrm{Mar}}$ & - & - & & & \\
\hline & $g(r)$ & $+0.8918 \pm .0947$ & $-0.2087 \pm .0146$ & $-0.2083 \pm .0071$ & & 0.69 \\
\hline & $g_{\mathrm{S}}(r)$ & $+0.7146 \pm .0785$ & $-0.2034 \pm .0140$ & $+0.2021 \pm .0063$ & & 0.69 \\
\hline & $f(r)$ & $+0.5706 \pm .0626$ & $-016069 \pm .0111$ & $+0.1597 \pm .0050$ & & $0 .{ }^{\prime} 69$ \\
\hline & $g(r)_{r_{0}=1.5 \mathrm{AU}}$ & $+2.7489 \pm .2280$ & $-0.0203 \pm .0129$ & $+0.0258 \pm .0136$ & & 0.68 \\
\hline \multirow[t]{6}{*}{$1991 \mathrm{~T} 2$} & $g(r)_{\mathrm{Mar}}$ & - & - & & & \\
\hline & $g^{*}(r) \mathrm{m}=-2.0$ & $+2.5159 \pm .5235$ & $-2.4769 \pm .4059$ & $+1.4182 \pm .3125$ & & $1^{\prime \prime} .14$ \\
\hline & $g^{*}(r) \mathrm{m}=-2.0, \mathrm{k}=0$ & $+1.9299 \pm .4339$ & $-1.4349 \pm .2211$ & $+0.7013 \pm .1754$ & & 1.' 13 \\
\hline & $g_{\mathrm{S}}(r)$ & $+2.4729 \pm .4927$ & $-1.9988 \pm .2951$ & $+1.0648 \pm .2298$ & & 1.' 13 \\
\hline & $f(r)$ & $+1.9137 \pm .3845$ & $-1.5436 \pm .2277$ & $+0.8118 \pm .1776$ & & 1.' 13 \\
\hline & $g(r)_{r_{0}=4 \mathrm{AU}}$ & $+2.8834 \pm .5592$ & $-2.3946 \pm .3535$ & $+1.3214 \pm .2744$ & & 1.' 13 \\
\hline \multirow[t]{4}{*}{1993 Y1 } & $g(r)_{\mathrm{Mar}}$ & +1.77 & +0.646 & & & \\
\hline & $g(r)$ & $+1.4777 \pm .1354$ & $-0.0204 \pm .0792$ & $-0.1103 \pm .0532$ & & $1 . .00$ \\
\hline & $g_{\mathrm{S}}(r)$ & $+1.4038 \pm .1197$ & $-0.0029 \pm .0657$ & $-0.0803 \pm .0449$ & & $1 . .00$ \\
\hline & $f(r)$ & $+1.0880 \pm .0955$ & $-0.0066 \pm .0542$ & $-0.0725 \pm .0370$ & & $1 . .00$ \\
\hline \multirow[t]{7}{*}{1995 O1 } & $g(r)_{\mathrm{Mar}}$ & +1.27 & +0.1144 & & & \\
\hline & $g(r)$ & $+1.520 \pm .031$ & $+0.1199 \pm .0053$ & $-0.0198 \pm .0117$ & & 1.. 07 \\
\hline & $g(r)$ & $+1.599 \pm .033$ & $-0.0425 \pm .0301$ & $-0.0361 \pm .0109$ & $8.23 \pm 1.46$ & $1 . .06$ \\
\hline & $g_{\mathrm{S}}(r)$ & $+1.4497 \pm .0339$ & $+0.1144 \pm .0052$ & $-0.0233 \pm .0107$ & & 1.' 10 \\
\hline & $g_{\mathrm{S}}(r)$ & $+1.5044 \pm .0367$ & $+0.0355 \pm .0218$ & $-0.0325 \pm .0103$ & $4.22 \pm 1.13$ & $1 . .09$ \\
\hline & $f(r)$ & $+1.147 \pm .027$ & $+0.0912 \pm .0043$ & $-0.0190 \pm .0086$ & & 1..' 10 \\
\hline & $f(r)$ & $+1.1845 \pm .0297$ & $+0.0384 \pm .0171$ & $-0.0253 \pm .0083$ & $3.55 \pm 1.11$ & 1.' 10 \\
\hline
\end{tabular}


Table 2. continued.

\begin{tabular}{|c|c|c|c|c|c|c|}
\hline Name & $\overline{h(r)}$ & $\overline{\overline{A_{1}}}$ & $\overline{\overline{A_{2}}}$ & $\overline{A_{3}}$ & $\tau$ [ [days] & rms \\
\hline \multirow[t]{3}{*}{1995 O1 cont. } & $g_{\mathrm{HB}}^{*}(r)$ & $+4.878 \pm .102$ & $+0.3802 \pm .0172$ & $-0.0670 \pm .0376$ & & 1..07 \\
\hline & $g_{\mathrm{HB}}^{*}(r)$ & $+5.093 \pm .110$ & $-0.0060 \pm .0877$ & $-0.1093 \pm .0356$ & $5.918 \pm 1.23$ & 1.'07 \\
\hline & $g(r)_{r_{0}=1.5 \mathrm{AU}}$ & $+1.4014 \pm .0276$ & $+0.1245 \pm .0059$ & $-0.0297 \pm .0142$ & & 1 ."06 \\
\hline \multirow[t]{5}{*}{$1990 \mathrm{~K} 1$} & $g(r)_{\mathrm{Mar}}$ & +3.0 & -0.2 & & & \\
\hline & $g(r)$ & $+3.3803 \pm .1059$ & $+0.1535 \pm .0518$ & $+0.3324 \pm .0147$ & & $1^{\prime \prime} .21$ \\
\hline & $g_{\mathrm{S}}(r)$ & $+2.7191 \pm .0793$ & $-0.0827 \pm .0450$ & $+0.2773 \pm .0122$ & & 1". 18 \\
\hline & $f(r)$ & $+2.2655 \pm .0671$ & $-0.0306 \pm .0365$ & $+0.2257 \pm .0099$ & & 1". 19 \\
\hline & $g(r)_{r_{0}=4 \mathrm{AU}}$ & $+3.1344 \pm .0929$ & $+0.0004 \pm .0493$ & $+0.3157 \pm .0138$ & & 1". 19 \\
\hline \multirow[t]{5}{*}{$1999 \mathrm{~J} 3$} & $g(r)_{\mathrm{Mar}}$ & - & - & & & \\
\hline & $g(r)$ & $+2.7344 \pm .1296$ & $-0.7626 \pm .0108$ & $-0.2305 \pm .0320$ & & 0.83 \\
\hline & $g_{\mathrm{S}}(r)$ & $+2.3812 \pm .1144$ & $-0.6330 \pm .0950$ & $-0.1843 \pm .0281$ & & 0.84 \\
\hline & $f(r)$ & $+1.8966 \pm .0910$ & $-0.5049 \pm .0756$ & $-0.1473 \pm .0223$ & & 0.84 \\
\hline & $g(r)_{r_{0}=1.5 \mathrm{AU}}$ & $+4.2603 \pm .1829$ & $-1.6007 \pm .1538$ & $+0.5491 \pm .0476$ & & 0.79 \\
\hline \multirow[t]{3}{*}{$1995 \mathrm{Y} 1$} & $g(r)_{\mathrm{Mar}}$ & +0.51 & -0.9335 & & & \\
\hline & $g^{*}(r) \mathrm{m}=-1.5, k=0$ & $+0.2781 \pm .1585$ & $-0.2374 \pm .1234$ & $+0.0806 \pm .0548$ & & 0.81 \\
\hline & $g(r)_{r_{0}=1.5 \mathrm{AU}}$ & $+1.3403 \pm .3293$ & $-0.9404 \pm .5659$ & $+0.1835 \pm .2937$ & & 0.81 \\
\hline \multirow[t]{8}{*}{1998 P1 } & $g(r)_{\mathrm{Mar}}$ & +31.51 & +2.2940 & & & \\
\hline & $g(r)$ & $+32.143 \pm .403$ & $+1.071 \pm .131$ & $-1.194 \pm .053$ & & $1{ }^{\prime \prime} 10$ \\
\hline & $g(r)$ & $+15.197 \pm .674$ & $-2.6934 \pm .1454$ & $-0.2870 \pm .0795$ & $56.4 \pm 4.3$ & 0.93 \\
\hline & $g_{\mathrm{S}}(r)$ & $+23.158 \pm .258$ & $+0.5434 \pm .1063$ & $-0.5934 \pm .0378$ & & 1.'02 \\
\hline & $g_{\mathrm{S}}(r)$ & $+12.384 \pm .514$ & $-1.7922 \pm .1380$ & $-0.0613 \pm .0765$ & $62.3 \pm 5.8$ & 0.92 \\
\hline & $f(r)$ & $+18.436 \pm .204$ & $+0.4392 \pm .0846$ & $-0.4686 \pm .0301$ & & $1{ }^{\prime \prime} 02$ \\
\hline & $f(r)$ & $+9.8560 \pm .4104$ & $-1.4270 \pm .1129$ & $-0.0440 \pm .0623$ & $62.7 \pm 5.9$ & 0.92 \\
\hline & $g(r)_{r_{0}=6 \mathrm{AU}}$ & $+24.382 \pm .267$ & $+0.5480 \pm .1141$ & $-0.5796 \pm .0400$ & & 1 .'01 \\
\hline \multirow[t]{4}{*}{1999 T1 } & $g(r)_{\mathrm{Mar}}$ & +3.52 & -1.5563 & & & \\
\hline & $g(r)$ & $+3.4457 \pm .1113$ & $-1.1405 \pm .0615$ & $-0.0976 \pm .0160$ & & 0.64 \\
\hline & $g_{\mathrm{S}}(r)$ & $+2.9990 \pm .0991$ & $-0.7838 \pm .0500$ & $-0.1057 \pm .0115$ & & 0.64 \\
\hline & $f(r)$ & $+2.4032 \pm .0796$ & $-0.6155 \pm .0397$ & $-0.0853 \pm .0090$ & & 0.64 \\
\hline \multirow[t]{4}{*}{1986 P1A } & $g(r)_{\mathrm{Mar}}$ & +1.8 & +0.1 & & & \\
\hline & $g(r)$ & $+1.7543 \pm .0687$ & $+0.0033 \pm .0032$ & $-0.0119 \pm .0096$ & & 1". 31 \\
\hline & $g_{\mathrm{S}}(r)$ & $+1.5054 \pm .0615$ & $-0.0682 \pm .0303$ & $-0.0151 \pm .0138$ & & 1'. 32 \\
\hline & $f(r)$ & $+1.2607 \pm .0513$ & $-0.0528 \pm .0255$ & $-0.0123 \pm .0118$ & & 1". 32 \\
\hline \multirow[t]{4}{*}{1996 E1 } & $g(r)_{\mathrm{Mar}}$ & - & - & & & \\
\hline & $g(r)$ & $+6.0250 \pm .9753$ & $+0.9554 \pm .4938$ & $-0.3074 \pm .3087$ & & 0.77 \\
\hline & $g_{\mathrm{S}}(r)$ & $+3.8164 \pm .6987$ & $+1.0348 \pm .3428$ & $-0.3378 \pm .2148$ & & 0.77 \\
\hline & $f(r)$ & $+3.3633 \pm .6088$ & $+0.8574 \pm .3002$ & $-0.2809 \pm .1879$ & & 0.77 \\
\hline \multirow[t]{4}{*}{$1998 \mathrm{~T} 1$} & $g(r)_{\mathrm{Mar}}$ & +0.59 & -0.3262 & & & \\
\hline & $g(r)$ & $+0.5919 \pm .0479$ & $-0.3180 \pm .0429$ & $-0.5651 \pm .0109$ & & 0.70 \\
\hline & $g_{\mathrm{S}}(r)$ & $+0.4474 \pm .0376$ & $-0.2441 \pm .0339$ & $-0.3614 \pm .0071$ & & 0.71 \\
\hline & $f(r)$ & $+0.3577 \pm .0301$ & $-0.1964 \pm .0273$ & $-0.2860 \pm .0056$ & & 0.71 \\
\hline \multirow[t]{4}{*}{$1975 \mathrm{~T} 1$} & $g(r)_{\mathrm{Mar}}$ & - & - & & & \\
\hline & $g(r)$ & $+4.6792 \pm .6797$ & $+1.1271 \pm .7851$ & $-1.4006 \pm .3853$ & & 1'. 31 \\
\hline & $g_{\mathrm{S}}(r)$ & $+3.2290 \pm .4686$ & $+0.4021 \pm .4178$ & $-0.8396 \pm .2071$ & & 1". 31 \\
\hline & $f(r)$ & $+2.5666 \pm .3729$ & $+0.2997 \pm .3282$ & $-0.6621 \pm .1627$ & & 1". 31 \\
\hline \multirow[t]{4}{*}{1987 W2 } & $g(r)_{\mathrm{Mar}}$ & - & - & & & \\
\hline & $g(r)$ & $+14.750 \pm 1.791$ & $+2.011 \pm .574$ & $-4.038 \pm .6062$ & & 1..06 \\
\hline & $g_{\mathrm{S}}(r)$ & $+8.165 \pm 1.025$ & $+0.8907 \pm .3574$ & $-2.0626 \pm .3376$ & & 1.'07 \\
\hline & $f(r)$ & $+6.4604 \pm .8122$ & $+0.6961 \pm .2843$ & $-1.626 \pm .2672$ & & 1..07 \\
\hline \multirow[t]{5}{*}{1993 A1 } & $g(r)_{\mathrm{Mar}}$ & +16.2 & -2.2 & & & \\
\hline & $g(r)$ & $+15.962 \pm .196$ & $-2.234 \pm .258$ & $+0.0507 \pm .0938$ & & 1". 14 \\
\hline & $g_{\mathrm{S}}(r)$ & $+5.8752 \pm .0780$ & $-0.1853 \pm .0937$ & $-0.0158 \pm .0295$ & & 1'. 11 \\
\hline & $f(r)$ & $+5.3268 \pm .0709$ & $-0.1550 \pm .0850$ & $-0.0147 \pm .0266$ & & 1.' 11 \\
\hline & $g(r)_{r_{0}=6 \mathrm{AU}}$ & $+6.7271 \pm .0913$ & $-0.1320 \pm .1074$ & $-0.0216 \pm .0334$ & & 1". 11 \\
\hline \multirow[t]{4}{*}{1991 B1 } & $g(r)_{\text {Mar }}$ & - & - & & & \\
\hline & $g(r)$ & $+7.8004 \pm .5799$ & $-1.0766 \pm .5990$ & $-0.0541 \pm .1539$ & & 0.93 \\
\hline & $g_{\mathrm{S}}(r)$ & $-0.8473 \pm .6729$ & $-0.0379 \pm .412$ & $-0.0784 \pm .251$ & & 0.92 \\
\hline & $f(r)$ & $+1.1027 \pm .0938$ & $-0.2946 \pm .1000$ & $+0.0060 \pm .0217$ & & 0.94 \\
\hline
\end{tabular}


M. Królikowska: Long-period comets with non-gravitational effects, Online Material p 5

Table 4. Forced precession model for assumed function $h(r)$ represented by: standard function $g(r), f(r), g^{*}(r)-$ modified function $g(r)$, respectively. NG parameters $A_{1}, A_{2}, A_{3}$ are given in units of $10^{-8} \mathrm{AU}$ day ${ }^{-2}$, and precession factor $f_{\mathrm{p}}$ - in units of $10^{6}$ day $\mathrm{AU}^{-1}$. Subscript " 0 " in $I_{0}$ and $\phi_{0}$ denotes the values on the starting epoch of integration given in the Col. 1. The $g^{*}(r)$ functions for C/1995 O1 Hale-Bopp $\left(g_{\mathrm{HB}}^{*}(r)\right)$ and C/1996 B2 Hyakutake $\left(g_{\mathrm{Hya}}^{*}(r)\right)$ are described in Table 3; subscripts ${ }^{(a)}$ and ${ }^{(b)}$ denote solutions taken from Szutowicz et al. (2002a,b). In the case of comet Hyakutake models with subscript ${ }^{(a)}$ additionally include the observed comet fragmentation on 1996 March 21.

\begin{tabular}{|c|c|c|c|c|c|c|c|}
\hline $\begin{array}{l}\text { Name } \\
\text { epoch }\end{array}$ & Model & $\overline{\bar{A}}$ & 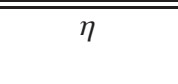 & $\overline{\overline{I_{0}}}$ & 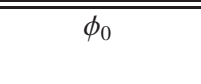 & $\begin{array}{c}f_{\mathrm{p}} \\
s\end{array}$ & rms \\
\hline 1996 B2 & $g(r)$ & $+3.1299 \pm .0485$ & $9.55 \pm 0.42$ & $65.41 \pm 0.89$ & $118.34 \pm 7.18$ & & 0,95 \\
\hline \multirow[t]{3}{*}{19960321} & $g_{\mathrm{Hya}}^{*}(r)$ & $+0.6457 \pm .0097$ & $13.02 \pm 1.22$ & $53.09 \pm 4.13$ & $23.16 \pm 3.13$ & & $0 ! 94$ \\
\hline & $g(r) \quad(a)$ & +4.2586 & 15.7 & 50.1 & 173.5 & $\begin{array}{c}+.25395 \\
+0.03\end{array}$ & 0.879 \\
\hline & $g_{\mathrm{Hya}}^{*}(r)^{(a)}$ & +4.8900 & 25.6 & 5.1 & 53.4 & $\begin{array}{c}-.80149 \\
-0.10\end{array}$ & 0.855 \\
\hline 1959 Y1 & $g(r)$ & $+5.6708 \pm .5026$ & $10.84 \pm 3.70$ & $172.8 \pm 13.8$ & 60.56 & & 1". 47 \\
\hline 195991218 & $f(r)$ & $+4.1079 \pm .3611$ & $10.72 \pm 3.92$ & $174.0 \pm 14.6$ & 69.74 & & 1." 47 \\
\hline $\begin{array}{l}1969 \text { Y1 } \\
19740404\end{array}$ & $g(r)$ & $+1.7101 \pm .1890$ & $9.21 \pm 4.71$ & $64.0 \pm 42.2$ & $110.2 \pm 72.3$ & & $1 " .53$ \\
\hline 1999 H1 & $g(r)$ & $+0.7313 \pm .1214$ & $30.50 \pm 6.45$ & $119.03 \pm 3.54$ & $338.43 \pm 4.90$ & & 0.69 \\
\hline 19990412 & $f(r)$ & $+0.4875 \pm .0800$ & $35.29 \pm 7.51$ & $119.26 \pm 3.44$ & $339.98 \pm 4.49$ & & 0.69 \\
\hline $1991 \mathrm{~T} 2$ & $g^{*}(r)$ & $+6.2412 \pm .8214$ & $58.78 \pm 2.82$ & $141.37 \pm 2.16$ & $123.49 \pm 5.33$ & & 1". 10 \\
\hline \multirow[t]{2}{*}{19910921} & $g^{*}(r) k=0$ & $+2.8437 \pm .4833$ & $43.30 \pm 3.67$ & $146.39 \pm 5.41$ & $124.0 \pm 15.8$ & & $1^{\prime \prime} 12$ \\
\hline & $f(r)$ & $+3.1034 \pm .4593$ & $47.95 \pm 3.38$ & $144.23 \pm 3.98$ & $123.9 \pm 10.6$ & & 1.' 11 \\
\hline 1993 Y1 & $g(r)$ & $+1.4714 \pm .1265$ & $6.08 \pm 3.84$ & $99.7 \pm 37.2$ & $164.6 \pm 63.3$ & & $1{ }^{\prime \prime} 00$ \\
\hline 19931129 & $f(r)$ & $+1.0832 \pm .0887$ & $5.83 \pm 4.18$ & $101.0 \pm 32.8$ & $176.4 \pm 57.9$ & & 1 ."00 \\
\hline 1995 O1 & $g(r)$ & $+1.5078 \pm .0307$ & $8.14 \pm 0.60$ & $55.62 \pm 3.01$ & $80.94 \pm 5.44$ & & 1."06 \\
\hline 20011018 & $g_{\mathrm{HB}}^{*}(r)^{(b)}$ & $+1.4281 \pm .0335$ & $7.44 \pm 0.88$ & $89.89 \pm 1.40$ & $70.09 \pm 1.51$ & $\begin{array}{l}-.0422 \\
-.30\end{array}$ & 1..06 \\
\hline $1990 \mathrm{~K} 1$ & $g(r)$ & $+3.7139 \pm .1226$ & $11.18 \pm 0.95$ & $60.21 \pm 3.49$ & $298.80 \pm 5.61$ & & $1^{\prime \prime} 20$ \\
\hline 19900529 & $f(r)$ & $+2.3215 \pm .0732$ & $8.14 \pm 0.50$ & $83.51 \pm 7.06$ & $323.25 \pm 7.41$ & & 1". 19 \\
\hline $1999 \mathrm{~J} 3$ & $g(r), f(r)$ & $+2.8491 \pm .1248$ & $24.50 \pm 4.10$ & $99.28 \pm 8.19$ & $90.84 \pm 2.39$ & & 0.80 \\
\hline \multirow[t]{2}{*}{20000406} & $g(r)$ & $+4.9549 \pm .6787$ & $-9.49 \pm 0.65$ & $63.97 \pm 5.60$ & $204.41 \pm 6.10$ & $\begin{array}{l}+.535 \pm 0.146 \\
+.707 \pm 0.038\end{array}$ & 0.78 \\
\hline & $g^{*}{ }_{\mathrm{Hya}}(r)$ & $+0.6806 \pm .0288$ & $11.19 \pm 1.26$ & $79.05 \pm 4.62$ & $75.48 \pm 4.69$ & $\begin{array}{l}+.5 \\
+.3\end{array}$ & 0.78 \\
\hline $\begin{array}{l}1995 \text { Y1 } \\
19951119\end{array}$ & $g^{*}(r)$ & $+0.8986 \pm .6687$ & $26.53 \pm 9.76$ & $69.4 \pm 37.2$ & $283.7 \pm 21.9$ & & 0.80 \\
\hline 1998 P1 & $g(r)$ & $+35.02 \pm .54$ & $6.23 \pm 0.66$ & $92.66 \pm 2.88$ & $91.79 \pm 2.64$ & & 1..06 \\
\hline \multirow[t]{2}{*}{19981103} & $f(r)$ & $+18.86 \pm .25$ & $2.60 \pm 0.46$ & $78.3 \pm 10.6$ & $108.0 \pm 11.6$ & & $1 . \prime 01$ \\
\hline & $g(r)$ & $+37.75 \pm .38$ & $8.94 \pm 0.38$ & $83.41 \pm 1.75$ & $129.42 \pm 0.78$ & $\begin{array}{l}-.2 \\
-.05\end{array}$ & 0.95 \\
\hline $1999 \mathrm{~T} 1$ & $g(r)$ & $+2.743 \pm .257$ & $26.47 \pm 4.90$ & $137.8 \pm 10.2$ & $285.36 \pm 2.73$ & & 0.'63 \\
\hline 19990919 & $f(r)$ & $+1.899 \pm .173$ & $19.54 \pm 2.98$ & $140.8 \pm 9.2$ & $285.12 \pm 4.94$ & & 0.63 \\
\hline 1987 W2 & $g(r)$ & $+13.82 \pm 2.28$ & $24.53 \pm 8.77$ & $72.01 \pm 8.19$ & $133.5 \pm 21.7$ & & 1."06 \\
\hline 19880320 & $f(r)$ & $+6.070 \pm 1.06$ & $22.62 \pm 8.90$ & $76.87 \pm 9.74$ & $133.6 \pm 23.0$ & & 1.'06 \\
\hline 1993 A1 & $g(r)$ & $+16.113 \pm .203$ & $8.19 \pm 1.11$ & $167.0 \pm 20.2$ & $94.3 \pm 15.4$ & & 1". 14 \\
\hline \multirow[t]{2}{*}{19921025} & $f(r)$ & $+5.2950 \pm .0730$ & $5.66 \pm 1.39$ & $106.61 \pm 9.88$ & $296.86 \pm 3.58$ & & 1". 10 \\
\hline & $f(r)$ & $+6.2176 \pm .3801$ & $3.44 \pm 1.33$ & $67.6 \pm 24.6$ & $285.17 \pm 3.63$ & $\begin{array}{l}+.307 \pm 0.348 \\
+.167 \pm 0.038 \\
\end{array}$ & 1.' 10 \\
\hline
\end{tabular}


Table 6. Comparison between two kinds of NG models for four short-period comets. NG parameters were derived from 8 of 12 detected apparitions for Comet 31P/Schwassmann-Wachmann 2, 8 of 9 detected apparitions for Comet 46P/Wirtanen (the last one were excluded), 15 of 19 apparitions for Comet 26P/Grigg-Skjellerup and all available observations (5 apparitions span over 9 perihelion passages) in the case of Comet 88P/Howell. Standard NG parameters $A_{1}, A_{2}, A_{3}$ and parameter $A$ (forced precession model) are given in units of $10^{-8} \mathrm{AU}$ day ${ }^{-2}$. The precession factor $f_{\mathrm{p}}$ is in units of $10^{7}$ day/AU, Subscript " 0 " in $I_{0}$ and $\phi_{0}$ denotes the values for the starting epoch of integration (Epoch: 200312 27). For two comets (31P and 46P) the assumption about the asymmetry of function $g(r)$ was necessary, and the time shifts $\tau$ (in days) were derived. The forced precession model of 26P was taken from Sitarski (2003).

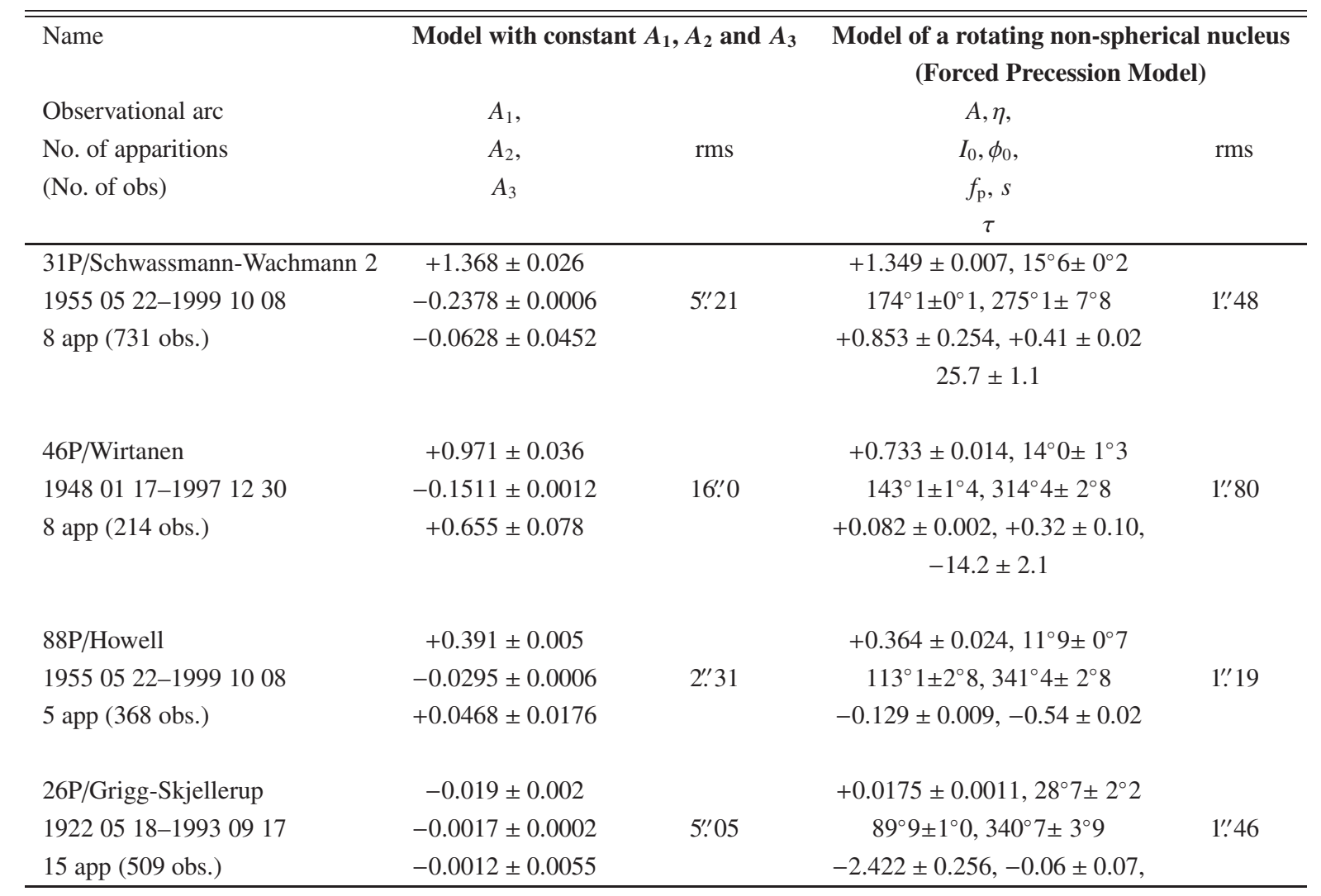

